\title{
异呋咱含能化合物合成及性能研究进展
}

\author{
薛 琪 $a, b$ 毕福强 $a, b$ 张家荣 $a, b$ 张俊林 $a, b$ \\ 王伯周*,a,b张生勇*, $a, c$ \\ ( ${ }^{a}$ 西安近代化学研究所 西安 710065) \\ ( ${ }^{b}$ 氟氮化工资源高效开发与利用国家重点实验室 西安 710065) \\ (c 空军军医大学药学系 西安 710032)
}

\begin{abstract}
摘要 异呋咱涵盖 1,3,4-噁二唑和 1,2,4-噁二唑, 是一类用于构成高能钝感含能材料的基本单元. 此类化合物已成为含 能材料领域的研究热点之一. 概述了两大类异呋咱含能化合物及其含能衍生物的最新研究进展, 着重探讨了异呋咱环 化反应机理和构建方法, 介绍了代表性异呋咱含能化合物的物化和爆轰性能, 并对其发展前景进行了展望.

关键词 异呋咱; 含能化合物; 合成; 爆轰性能
\end{abstract}

\section{Advances in the Synthesis and Properties of the Isofurazan Energetic Compounds}

\author{
Xue, $\mathrm{Qi}^{a, b}$ \\ Bi, Fuqiang ${ }^{a, b}$ \\ Zhang, Jiarong ${ }^{a, b}$ \\ Zhang, Junlin \\ Wang, Bozhou*,a,b \\ Zhang, Shengyong *,a,c \\ ( ${ }^{a}$ Xi'an Modern Chemistry Research Instritute, Xi'an 710065) \\ ( ${ }^{b}$ State Key Laboratory of Fluorine \& Nitrogen Chemical, Xi'an 710065) \\ ( ${ }^{c}$ School of Pharmacy, Fourth Military Medical University, Xi'an 710032)
}

\begin{abstract}
Isofurazan is composed of 1,3,4-oxadiazole and 1,2,4-oxadiazole, and the isofurazan compounds have gained considerable prominence as energetic compounds. This review focues on the two major classes of isofurazan energetic compounds and their energetic derivatives. The cyclization methods and reaction mechanism of isofurazans are presented. Several energetic compounds of isofurazans with excellent performances are highlighted, and their main physicochemical properties and detonation performances and their development prospect are discussed.

Keywords isofurazan; energetic compound; synthesis; detonation performances
\end{abstract}

近几十年, 高能量密度材料的(HEDMS)的发展十 分迅速, 追求高能量成为含能材料永恒的目标之一. 随 着武器弹药使用环境的日益苛刻, 在追求弹药高能量的 同时, 对其安全性的要求也越来越高. 如何实现高能与 安全的平衡, 设计和合成出高能、高氧平衡、低感且环 境友好新型含能材料一直是研究人员的不解追求 ${ }^{[1 \sim 6]}$.

富氮五元杂环是常见的含能材料母体环, 其中, 1,2,5-噁二唑环(呋咱环)因有着优良的性能而得到深入 研究: 1,2,5-呋咱环含有两个 $\mathrm{C}=\mathrm{N}$ 和两个 $\mathrm{N}-\mathrm{O}$ 键, 为 其提供了高生成焓和高氧平衡值 ${ }^{[8,9]}$, 呋咱的芳香型平
面结构增加了其结构的稳定性, 近年来, 在这一领域取 得了显著进展，合成了一系列性能优异的含能化合 物 ${ }^{[9 \sim 15]}$. 异呋咱是呋咱的同分异构体, 涵盖 1,2,4-噁二 唑和 1,3,4-噁二唑两种氮杂五元环, 但生成焓远低于呋 咱 ${ }^{[16]}$, 先前很少将它们作为含能材料的母体单元进行 研究. 2012 年, 陈甫雪等 ${ }^{[17]}$ 首次报道了以 1,2,4-啞二唑 为母体环的一系列含能化合物, 其中 3-硝基-5-硝氨基1,2,4-惡二唑的理论密度为 $1.883 \mathrm{~g} \cdot \mathrm{cm}^{-1}$, 理论爆速 9095 $\mathrm{m} \cdot \mathrm{s}^{-1}$, 理论爆压 $37.68 \mathrm{GPa}$, 而其摩擦感度仅为 $15 \mathrm{~J}$, 与 TNT 相当, 是一种新型高能针感含能材料. 从此, 异呋

\footnotetext{
* Corresponding authors. E-mail: wbz600@163.com; syzhang@fmmu.edu.cn Received November 23, 2018; revised January 13, 2019; published online January 31, 2019. Project supported by the National Natural Science Foundation of China (No. 21243007). 国家自然科学基金(No. 21243007)资助项目.
} 
咱类含能材料逐渐受到科研人员的关注并迅速发展, 成 为含能材料合成领域的研究热点之一.

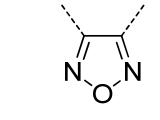

1,2,5-oxadiazole $216.3 \mathrm{~kJ} \cdot \mathrm{mol}^{-1}$

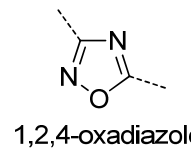

$99.8{\mathrm{~kJ} \cdot \mathrm{mol}^{-1}}^{-1}$

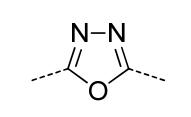

1,3,4-oxadiazole $72.2 \mathrm{~kJ} \cdot \mathrm{mol}^{-1}$
图 1 呋咱与异呋咱的结构及生成焓 ${ }^{[7]}$

Figure 1 Structures, and enthalpies of formation of furazan and isofurazan

异呋咱环虽然生成焓较低, 但稳定性远高于呋咱, 可以以其作为母体结构, 再引入高能化学基团(如呋咱 环、 $\left.\mathrm{NH}_{2} 、 \mathrm{NO}_{2} 、 \mathrm{ONO}_{2} 、 \mathrm{~N}_{3} 、 \mathrm{NHNO}_{2}\right)^{[18 \sim 19]}$, 有效地提 高化合物的生成焓, 通过氮杂骨架与含能基团的调控可 获得性能优良的含能材料. 依据这研究思路, 目前已合 成部分性能优异的异呋咱含能化合物, 如理论爆速大于 $10000 \mathrm{~m} \cdot \mathrm{s}^{-1}$ 高能单质炸药 $\mathrm{DDAzF} \mathrm{F}^{[20]}$, 高能钝感耐热炸 药 TKX-55 ${ }^{[21]}$, 可作为高能增塑剂的 5,5'-乙硝酸酯- 3,3'联-1,2,4-噁二唑 ${ }^{[22]}$ 等.

第二种研究思路是, 将异呋咱环作为降感基团引入 已知高能含能化合物中，与其芳香性环形成共轭体系， 有望实现含能材料高能与安全性的矛盾与统一. 以 3,4二(2,2,2-三硝基乙氨基)-呋咱 ${ }^{[23]}$ 为例，其爆速达 8553 $\mathrm{m} \cdot \mathrm{s}^{-1}$, 撞击感度(IS)约为 $8 \mathrm{~J}$, 分解温度 $156{ }^{\circ} \mathrm{C}$; 如将其 单侧的 2,2,2-三硝基乙氨基替换为 5-甲基-1,2,4-噁二 唑 ${ }^{[24]}$, 爆速为 $8118 \mathrm{~m} \cdot \mathrm{s}^{-1}$, 摩擦感度 $(\mathrm{FS})>360 \mathrm{~N}, \mathrm{IS}>$ $60 \mathrm{~J}$, 其分解点温度升高, 机械感度大幅降低, 表明 $1,2,4$-惡二唑是设计、合成不敏感含能材料的理想单元 结构(图 2).
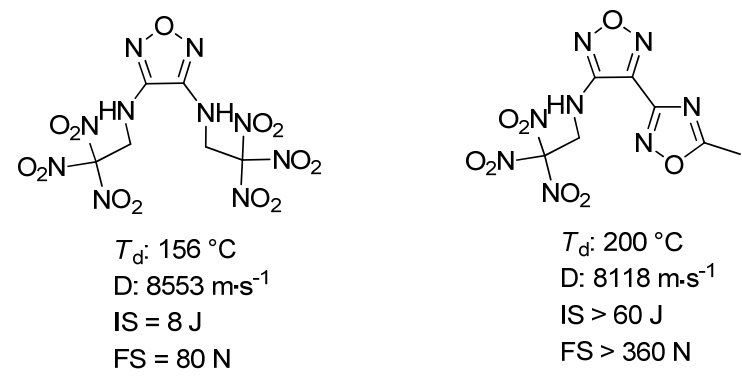

图 2 异呋咱的引入对爆轰性能的影响

Figure 2 Effect of introduction for isofurazan on detonation performances

由于异呋咱含能化合物研究时间不长, 对异呋咱含 能材料环母体合成方法和合成机理的综述还尚未见报 道, 而这方面内容的总结对于异呋咱含能化合物的进一 步发展很有必要. 因此, 本文以目前合成的异呋咱含能 化合物为研究背景, 着重介绍了 1,2,4-噁二唑及 1,3,4-噁
二唑等氮杂五元环的构建方法，探讨了环化反应机理; 全面概述了近年来异呋咱含能化合物的最新合成研究 进展，并对典型含能化合物的物化、爆轰性能进行了阐 述，以期为进一步开展相关研究工作提供参考.

\section{1,2,4-噁二唑含能化合物的合成及性能}

\section{1 偕胺肟与羧酸衍生物合成 1,2,4-噁二唑}

以偕胺肟与羧酸衍生物为底物合成 1,2,4-噁二唑含 能化合物是最常见的构建方法. 反应过程可概括为: 偕 胺肜与活化的羧酸衍生物发生 $O$-酰化反应得到 $O$-酰化 中间体，然后进行脱水环化.

\subsection{1 偕胺肟与酰氯合成 $1,2,4-$ 噁二唑}

酰氯反应活性高, 酰氯与偕胺肟的 $O$-酰化易于进 行. Nishiwaki 等 ${ }^{[25]}$ 发现采用有机碱(吡啶、三乙胺)作为 缚酸剂, 11 种芳香族或脂肪酰氯与偕胺肟在室温下摚拌 隔夜即可生成 $O$-酰化中间体(Eq. 1).

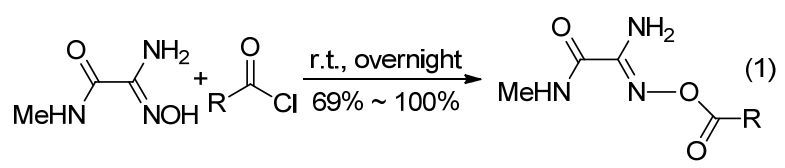

酰氯与偕胺肜 $O$-酰化反应(以吡啶作缚酸剂为例) 机理见如图 3.

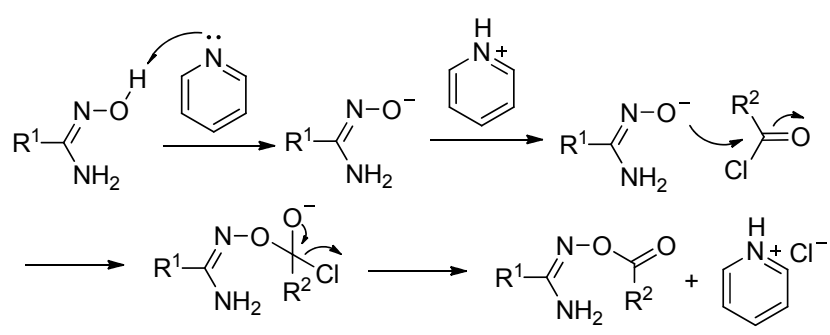

图 3 酰氯与偕胺肜 $O$-酰化反应机理

Figure 3 Proposed $O$-acylation reaction mechanism of acylchloride and amidoximes

相较于酰氯的 $O$-酰化反应，其 $O$-酰化中间体的脱 水环化是反应的决速步骤 ${ }^{[26]}$. 主要的脱水方法有加热 法、碱催化法、微波辐射法等.

加热法是指 $O$-酰化中间体在高温下 $\left(100{ }^{\circ} \mathrm{C}\right.$ 以上 $)$ 长时间反应脱水得到环化产物 ${ }^{[27,28]}$, 这种方法选择性 差，反应时间长，收率低. 其可能的反应机理见图 $4^{[29]}$.

碱催化法是目前研究较多的方法, 碱催化可加快反 应进程，可能的反应机理见图 5 .

反应一般采用吡啶、三乙胺等有机弱碱作为催化剂. Shine 等 ${ }^{[30]}$ 采用 15 种脂肪族或苯基酰氯与 5 种苯基或芳 香族偕胺肜在吡啶中回流下反应 $30 \mathrm{~min}$, 即可得到 1,2,4-噁二唑(Eq. 2). 


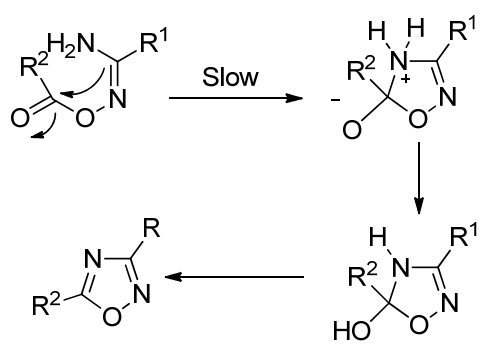

图 $4 O$-酰化中间体加热环化机理

Figure 4 Proposed heating cyclization mechanism of $O$-acylation intermediate

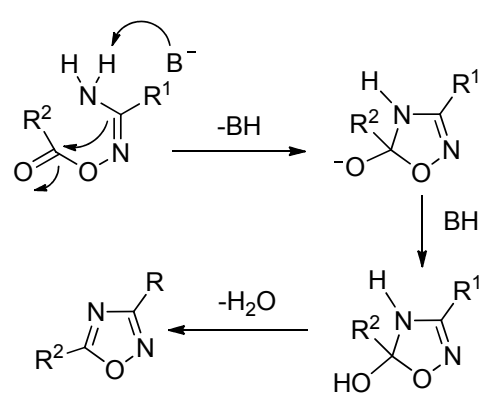

图 5 O-酰化中间体碱催化环化机理

Figure 5 Proposed cyclization mechanism of O-acylation intermediate via base catalysis

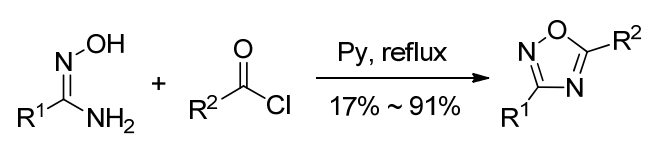

Gangloff 等 ${ }^{[31]}$ 首次报道了以 $O$-酰化中间体作为底 物, 在室温下 $\mathrm{THF} /$ 四丁基氟化铵(TBAF)体系中合成 3,5 取代的 1,2,4-噁二唑. 环化反应收率受取代基影响较大, 延长反应时间不能提高收率(Eq. 3).

$$
\prod_{\mathrm{R}^{1}}^{\mathrm{R}^{2}} \underset{\mathrm{NH}_{2}}{\mathrm{O}_{\mathrm{N}}} \frac{\mathrm{Bu}_{4} \mathrm{NF}, \mathrm{THF}, 23^{\circ} \mathrm{C}}{5 \% \sim 98 \%} \underset{\mathrm{R}^{1}}{\stackrel{N}{N}} \stackrel{\mathrm{O}}{\mathrm{N}^{-\mathrm{R}^{2}}}
$$

Nishiwaki 等 ${ }^{[25]}$ 以(Z)-2-乙酰氧基-2-氨基- $N$-甲基乙 酰胺为原料, 比较了吡啶、三乙胺、 $\mathrm{TBAF}$ 作为催化剂 时环化产物的收率. 用 1 equiv.三乙胺(Eq. 4)和吡啶(Eq. 5)在 $80{ }^{\circ} \mathrm{C}$ 加热 $2 \mathrm{~h}, 1,2,4$-惡二唑收率仅为 $12 \%$ 和 $25 \%$; 用 0.1 equiv. TBAF 处理原料, 在室温下反应 $2 \mathrm{~h}$ 即可以 $100 \%$ 的收率得到产品(Eq. 6).

氟阴离子具有腐蚀性, 目前已提出了一些替代环化

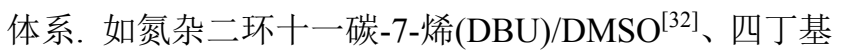
氢氧化铵 $(\mathrm{TBAH}) / \mathrm{DMSO}^{[33]} 、 \mathrm{KOH} / \mathrm{DMSO}$ 等. 这些体系 的特点是反应时间短, 室温下即可完成环化反应.

微波合成是近十年来发展最快的方法, 其优点为反 应时间更快，产率更高，副产物含量少. Kaboudin 等 ${ }^{[34]}$

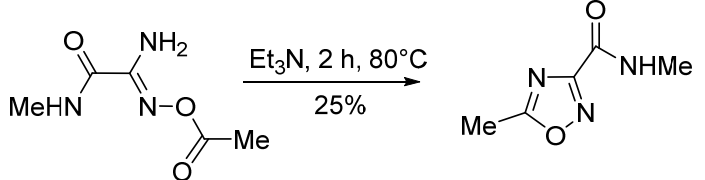<smiles>CNC(=O)/C(N)=N/OC(C)=O</smiles><smiles>CNC(=O)c1noc(C)n1</smiles><smiles>CNC(=O)/C(N)=N/OC(C)=O</smiles><smiles>CNC(=O)c1noc(C)n1</smiles>

报道了在微波/ $\mathrm{MgO}$ 催化作用下，10 种不同的苯基偕胺 肟与酰氯仅反应 $1 \mathrm{~min}$ 就可以高收率地得到 1,2,4-噁二 唑(67\% 91\%) (Eq. 7).

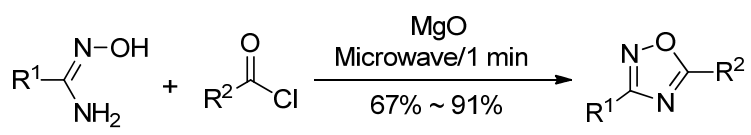

在有机合成中，微波技术使得反应热效应及加热效 率大大增加, 但难以控制反应进度, 在含能化合物的合 成中应用较少.

1990 年 Kiseleva 等 ${ }^{[35]}$ 首次报道了 3,3'-联-1,2,4-噁二 唑-5,5'-乙二酸二乙酯的合成(Eq. 8), 但该合成方法选择 性较差, 操作较为复杂, 收率较低. 2017 年黄晓川等 ${ }^{[36]}$ 报道了它的改进方法(Eq. 9), 利用氯甲酰乙酸乙酯中高 活性的氯甲酰基替代酯基，在较低的温度下与二氨基乙 二肟发生缩合环化反应，与原反应相比，降低了反应温 度，提高了反应的选择性.
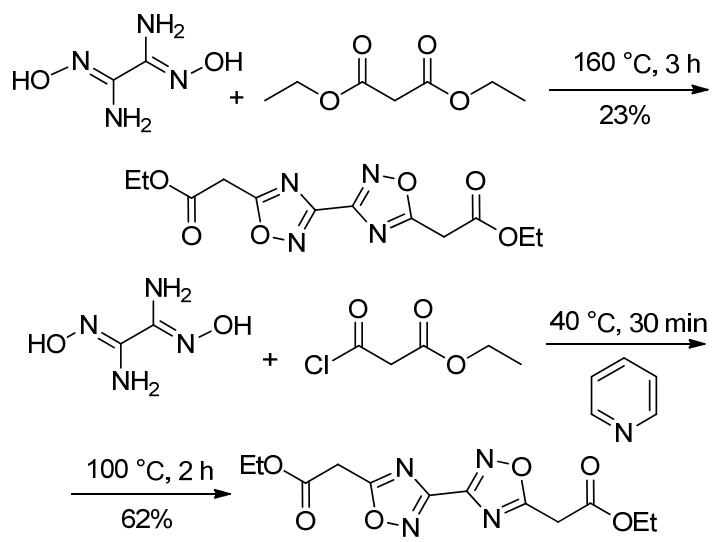

3,3'-联-1,2,4-噁二唑-5,5'-乙二酸二乙酯是重要的含 能化合物中间体, 它具有芳香共平面结构, 偶极矩小, 结构较为紧凑, 因此能有效提高化合物的密度及稳定 性 ${ }^{[37]}$. 此外, 利用乙酸乙酯这一反应活性位点, 通过硝 
化、氟化等反应，可将一些高能基团引入酯的 $\alpha$-碳上.

Klapötke 课题组 ${ }^{[7]}$ 以 3,3'-联-1,2,4-噁二唑-5,5'-乙二 酸二乙酯(1)为原料, 经硝化得到 3,3'-联-1,2,4-噁二唑5,5'-二硝基乙二酸二乙酯(2), 在碱性条件下脱酯形成偕 二硝基阴离子, 随后与含能阳离子配位得到 10 种含能 离子盐 $(\mathbf{2} \mathbf{a} \sim \mathbf{2 j})$. 其中羟胺盐 $\mathbf{2 h}$ 的理论密度达 $1.946 \mathrm{~g} \cdot$ $\mathrm{cm}^{-3}(298 \mathrm{~K})$, 理论爆速达 $8935 \mathrm{~m} \bullet \mathrm{s}^{-1}$, 而且其撞击感度 为 $4 \mathrm{~J}$, 摩擦感度为 $108 \mathrm{~N}$, 是一种具有潜在应用价值的 高能含能离子盐(图 6).

此外, 他们以铵盐为原料, 经氟化反应成功引入了 氟二硝基甲基得到 5,5'-氟二硝基甲基-3,3'-联-1,2,4-噁二 唑(3) ${ }^{[38]}$; 经硝化反应, 引入三硝甲基得到 5,5'-三硝甲 基-3,3'-联-1,2,4-噁二唑(4) ${ }^{[39]} .3$ 的计算密度为 $1.96 \mathrm{~g} \bullet$ $\mathrm{cm}^{-3}(298 \mathrm{~K})$, 理论爆速达 $8367 \mathrm{~m} \cdot \mathrm{s}^{-1}$, 撞击感度为 $10 \mathrm{~J}$, 摩擦感度为 $192 \mathrm{~N}$, 是一种潜在的不敏感含能材料; 4 的 计算密度为 $1.94 \mathrm{~g} \bullet \mathrm{cm}^{-3}(298 \mathrm{~K})$, 理论爆速达 $8814 \mathrm{~m} \cdot$ $\mathrm{s}^{-1}$, 氧平衡水平 $\Omega_{\mathrm{CO} 2}$ 为 $7.3 \%$, 一种综合性能优良的氧 化剂(图 7).

Gregory 等 ${ }^{[40]}$ 首次报道了 $1,2,4$-噁二唑-3,5-二甲酸 乙酯的合成, 采用氨基肜甲酸乙酯与氯甲酰乙酸乙酯在 $\mathrm{CHCl}_{3}$ 中(吡啶催化)下首先发生 $O$-酰化反应, 然后回流 环化，收率达 99\%以上(Eq. 10).

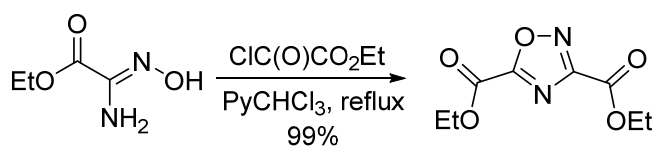

Pagoria 等 ${ }^{[41]}$ 采用这一反应前体侧链的酯基经酰氨 化、脱水转变为氰基，氭基再经肜化、氯化、叠氮化以 及环化生成 3,5-二(2-羟基-四唑基)-1,2,4-噁二唑(7). 该 含能化合物显酸性, 在氨气的乙醇溶液中发生中和反应 生成含能铵盐(7a), 但其含能离子盐的能量水平及稳定 性均未见报道(图 8).<smiles>CCOC(=O)Cc1nc(-c2noc(CC(=O)OCC)n2)no1</smiles><smiles>CCOC(=O)C(C(=O)OCC)(c1nc(-c2noc(C(O)([N+](=O)[O-])C(N)(C(=O)OCC)C(=O)OCC)n2)no1)[N+](=O)[O-]</smiles>
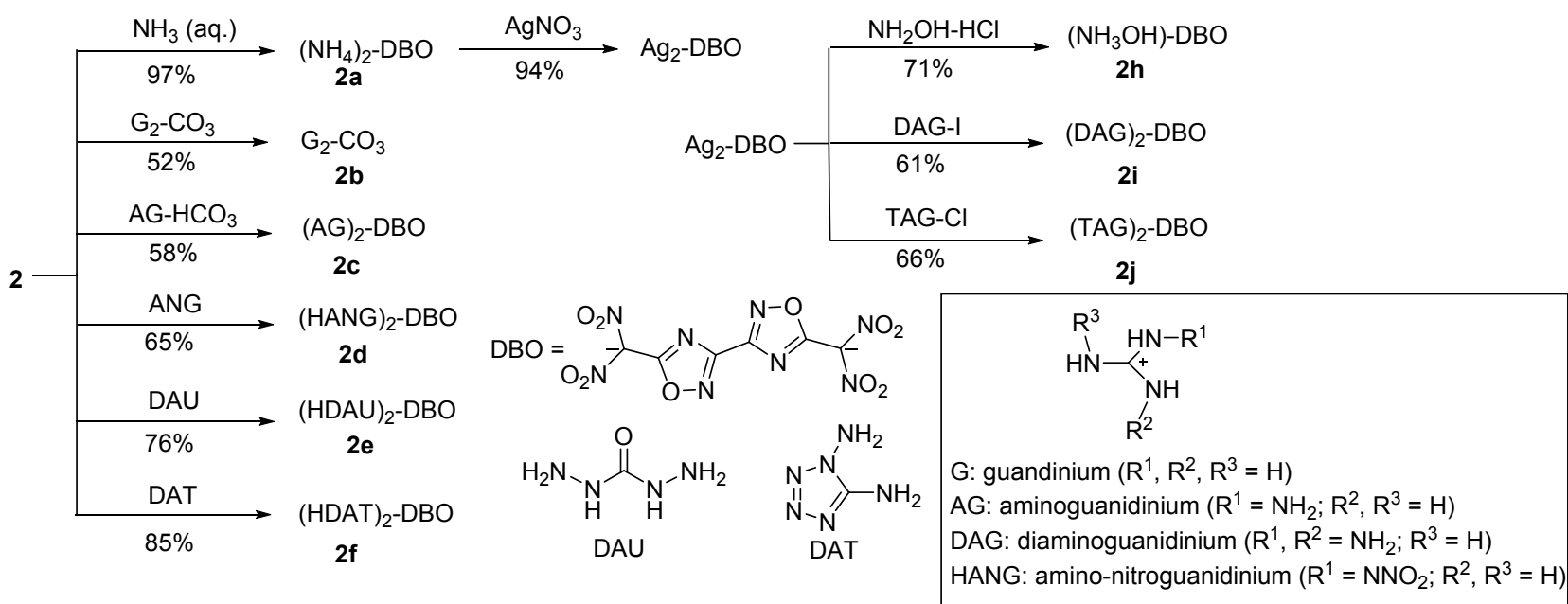

图 6 化合物 2 及其含能离子盐的合成

Figure 6 Synthesis of compound 2 and its energetic ion salts
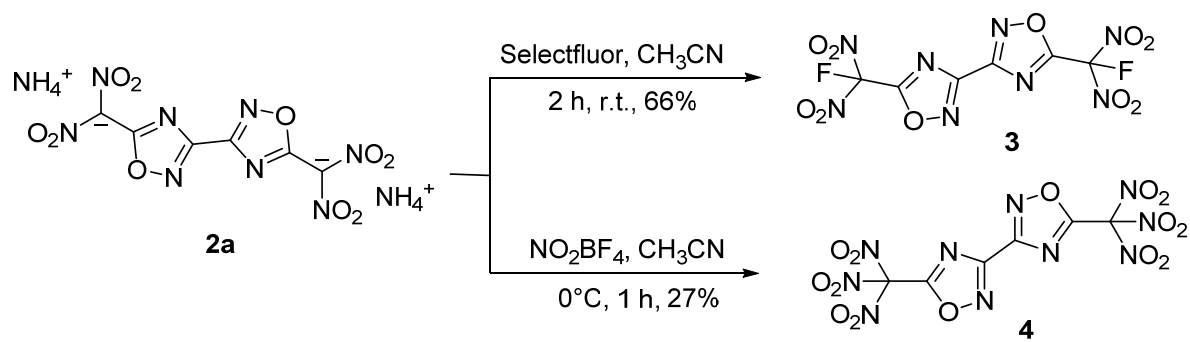

图 7 化合物 3 及 4 的合成

Figure 7 Synthesis of compounds 3 and 4 
<smiles>CCOC(=O)c1noc(C(=O)OCC)n1</smiles>

(1) $\mathrm{NH}_{3}$ in $\mathrm{MeOH}$, r.t.

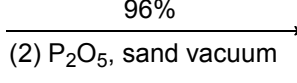

$210{ }^{\circ} \mathrm{C}$ liq. $\mathrm{N}_{2}$ trap, $70 \%$

$\underset{\substack{\mathrm{NC} \\ 6}}{\mathrm{~N}}$

(1) $\mathrm{H}_{2} \mathrm{NOH} \mathrm{HCl}$

$\mathrm{KOH} \mathrm{MeOH}, 73 \%$

(2) $\mathrm{HCl} \mathrm{NaNO} 2,87 \%$

(3) $\mathrm{NaN}_{3}, \mathrm{DMF}, 78 \%$

(4) $\mathrm{HCl}$, dioxane, $\mathrm{Et}_{2} \mathrm{O}$ $87 \%$<smiles></smiles>

$\frac{\mathrm{NH}_{3} \text { (aq.) EtOH, } \mathrm{H}_{2} \mathrm{O}}{72 \%}$<smiles>[NH3+]On1nnnc1-c1noc(-c2nnnn2O)n1</smiles>

$7 a$

图 8 化合物 7 及其含能铵盐 $7 \mathbf{a}$ 的合成

Figure 8 Synthesis of compound 7 and its energetic ion salt 7a

Sabatini 等 ${ }^{[22]}$ 以二氨基乙二肟和乙酰氧基乙酰氯为 原料, 经脱水环化、水解、硝化反应得到化合物 3,3'-联1,2,4-貿二唑-5,5'-二乙硝酸酯(10), 其理论密度达 1.832 $\mathrm{g} \cdot \mathrm{cm}^{-3}$, 爆速 $8180 \mathrm{~m} \bullet \mathrm{s}^{-1}$, 熔点 $84.5{ }^{\circ} \mathrm{C}, T_{\mathrm{dec}}=183.5{ }^{\circ} \mathrm{C}$, IS 为 $8.7 \mathrm{~J}, \mathrm{FS}$ 为 $282 \mathrm{~N}$.
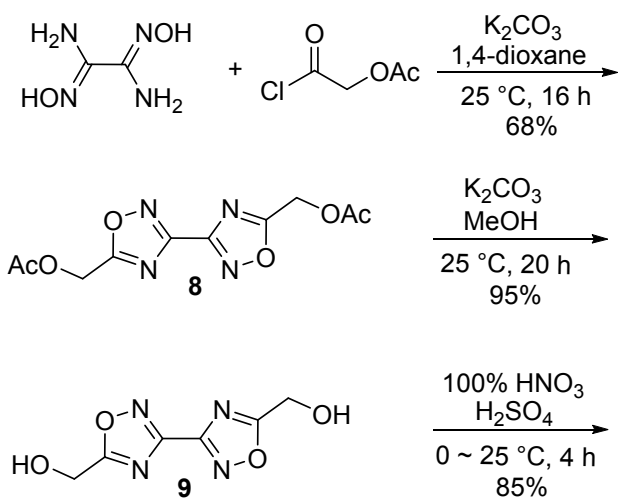<smiles>O=[N+]([O-])Cc1nc(-c2noc(CO[N+](=O)[O-])n2)no1</smiles>

图 9 化合物 $\mathbf{1 0}$ 的合成

Figure 9 Synthesis of compound $\mathbf{1 0}$

Pagoria 等 ${ }^{[41]}$ 利用二氨基乙二肜与草酰氯单乙酯脱 水环化合成 3,3'-联-1,2,4-惡二唑-5,5'-二甲酸乙酯(11), 经酰氨化、脱水反应将酯基变为氰基，氧基再经肜化、 氯化、叠氮化以及环化生成羟基四唑(13). 该化合物在 碱性条件下与盐酸着弪胺进行离子交换获得羟胺含能离 子盐(13a).

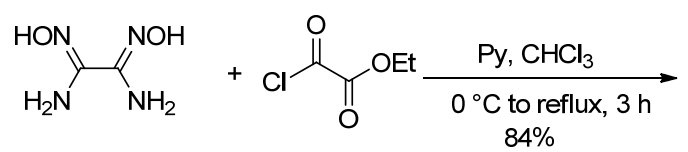<smiles>CCOC(=O)c1nc(-c2noc(C(=O)OCC)n2)no1</smiles>

(1) $\mathrm{NH}_{3}$ in $\mathrm{MeOH}$

$\underset{\text { (2) TFAA, Py, 1,4-dioxane }}{\stackrel{\text { r.t. } 16 \text { h, } 92 \%}{ }}$ $0{ }^{\circ} \mathrm{C}$ to r.t., $80 \%$

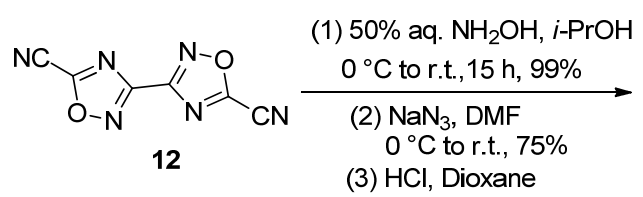
$40^{\circ} \mathrm{C}, 20 \mathrm{~h}, 77 \%$
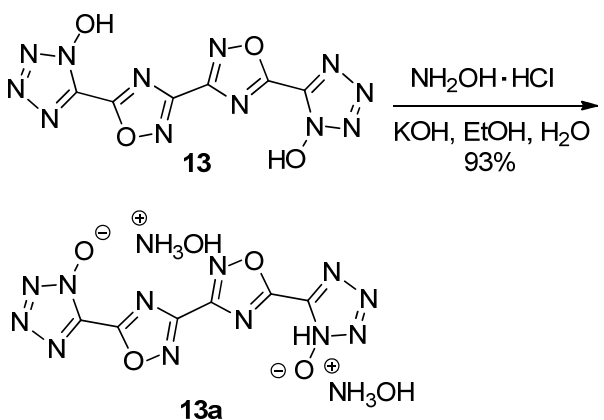

图 10 化合物 13 及其含能离子盐的合成

Figure 10 Synthesis of compound $\mathbf{1 3}$ and its energetic ion salt

\subsection{2 偕胺肜与酯合成 $1,2,4-$ 噁二唑}

酯与偕胺肜可经 $O$-酰化和脱水环化合成 1,2,4-噁二

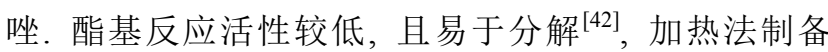
1,2,4-噁二唑的收率很低. 在碱催化下，该反应收率可 大大提高：在碱的存在下芳基肟的氧原子上快速形成共 振稳定的阴离子，帮助亲核进攻反应的进行. 反应机理 如图 11 所示.
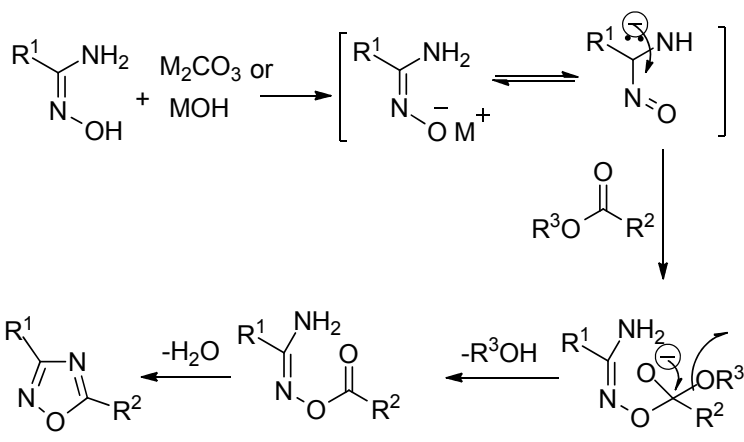

图 11 偕胺肜与酯的脱水环化反应机理

Figure 11 Proposed dehydration and cyclization mechanism of amidoximes and ester

Srivastava 等 ${ }^{[43]}$ 利用 $\mathrm{K}_{2} \mathrm{CO}_{3}$ 作为 $O$-酰化反应的活化 剂，用 2-羟基乙酸乙酯和不同取代基苯基偕胺肜在甲 
苯/DMF $(V: V=9: 1)$ 的混合溶剂中回流 $8 \mathrm{~h}$, 脱水环化 得到 1,2,4-噁二唑，产率为 $35 \% \sim 76 \%$ (Eq. 11).

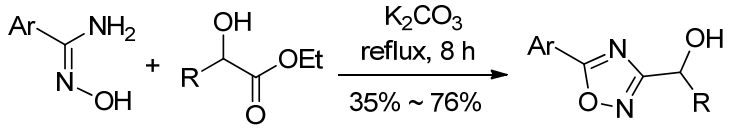

每一个呋咱环可将含能化合物的密度提高 0.06 $0.08 \mathrm{~g} \cdot \mathrm{cm}^{-3}$ ，爆速增加 $300 \mathrm{~m} \cdot \mathrm{s}^{-1[44]}$. 计算结果证实，分 子中引入不同的啞二唑环的是一种有效的降低感度并 获得良好爆轰性能的方法 ${ }^{[45]}$. Kuklja 等 ${ }^{[46]}$ 以 4-氨基1,2,5-呋咱-3-偕胺肟及草酸二乙酯为原料, 以高位阻有 机碱 2,4,6-三甲基吡啶为催化剂, 脱水环化得化合物 DATO (15), 再经硝化得 LLM-200 (16). LLM-200 理论 密度达 $1.940 \mathrm{~g} \bullet \mathrm{cm}^{-3}$, 爆速达 $8780 \mathrm{~m} \bullet \mathrm{s}^{-1}$, 熔点 $180{ }^{\circ} \mathrm{C}$, $T_{\mathrm{dec}}=295{ }^{\circ} \mathrm{C}$, 特性落高 $H_{50}=62 \mathrm{~cm}$, 可见 LLM-200 是 一种爆轰性能与 RDX 相当, 稳定性好的高能钝感耐热 炸药.

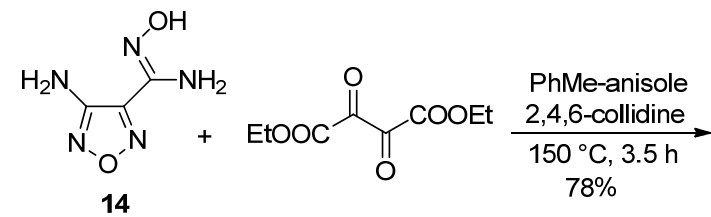<smiles>Nc1nonc1-c1noc(-c2nc(-c3nonc3N)no2)n1</smiles><smiles>O=[N+]([O-])c1nonc1-c1noc(-c2nc(-c3nonc3[N+](=O)[O-])no2)n1</smiles>

图 12 化合物 LLM-200 (16)合成

Figure 12 Synthesis of compound LLM-200 (16)

\subsection{3 偕胺肜与酸酎合成 $1,2,4$-噁二唑}

酸酐是很强的酰化剂, 易于和偕胺肪发生 O-酰化 反应. 该反应可同时被酸催化和碱催化. 在酸催化下, 酸酐与偕胺肜的酰化反应机理如图 13 所示.<smiles>[R]C(=O)[R7]C(=O)OCCCCC([R])OC([R])=O</smiles><smiles>[R]C(=O)[R]C(N)=NOC([R])=O</smiles>

图 13 偕胺肜与酸酐的 $O$-酰化反应机理(酸催化)

Figure 13 Proposed $O$-acylation mechanism of amidoximes and anhydride via acid catalysis
在碱催化下，酸䣶与偕胺肜的酰化反应机理如图 14 所示(以吡啶为例).<smiles>[R]C(=O)OC(=O)CCCC[O+]N=C([R])N</smiles>

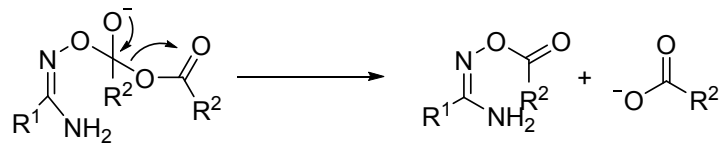

图 14 偕胺肜与酸酐的 $O$-酰化反应机理(碱催化) Figure 14 Proposed $O$-acylation mechanism of amidoximes and anhydride via base catalysis

Baykov 等 ${ }^{[47]}$ 报道了 21 种芳香型偕胺肟与环内酸酐 的 $O$-酰化反应，在 DMSO 中室温反应 $1 \mathrm{~h}$ 便可以高收率 地得到酰化产物, 酰化产物在 $\mathrm{NaOH}$ 催化下反应即可发 生脱水成环(Eq. 12).

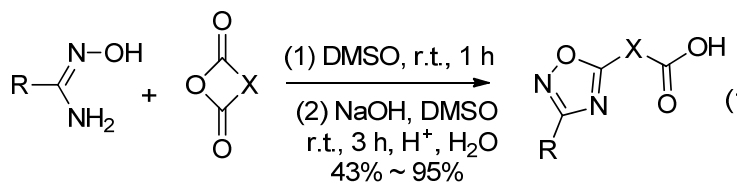

Vidavalur 等 ${ }^{[48]}$ 报道了以二氧化硅负载的高氯酸 $\left(\mathrm{HClO}_{4}-\mathrm{SiO}_{2}\right)$ 作为催化剂, 在无溶剂条件下, 芳香型偕 胺肟与 7 种酸酐一锅法制备 1,2,4-噁二唑，反应时间短， 收率高(Eq. 13).

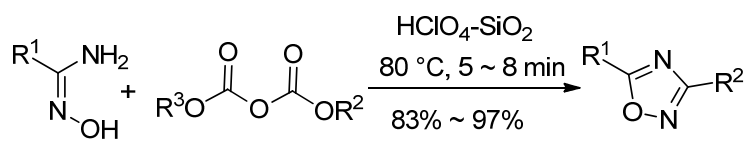

Klapötke 等 ${ }^{[38]}$ 报道了 5,5'-三氟甲基-3,3'-联-1,2,4-噁 二唑的合成. 三氟乙酸酐与二氨基乙二肜在 $35{ }^{\circ} \mathrm{C}$ 下反 应 $3 \mathrm{~h}$ 即可得到产品(Eq. 14).

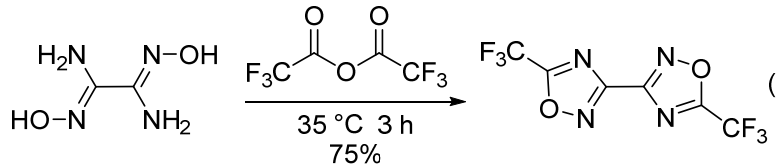

联-1,2,4-噁二唑具有结构稳定、紧凑的特点, 三氟 甲基是非能量基才，但可以提高化合物的密度．该化合 物的密度达 $2.01 \mathrm{~g} \cdot \mathrm{cm}^{-3}$. 熔点为 $98{ }^{\circ} \mathrm{C}$, 分解温度为 $142{ }^{\circ} \mathrm{C}$. 此外, 氟原子可以被硝基逐步取代 ${ }^{[37,38]}$, 有望 得到新型含能材料.

$\mathrm{Qu}$ 等 ${ }^{[20]}$ 以 3-氨基-4-偕胺肜基呋咱(17) (图 15)为原 料，分别与乙酸酐、三氟醋酐反应，经 $O$-酰化环化得到 1,2,4-噁二唑，最后分别在 $\mathrm{KMnO}_{4} / \mathrm{HCl}$ 体系中反应得到 


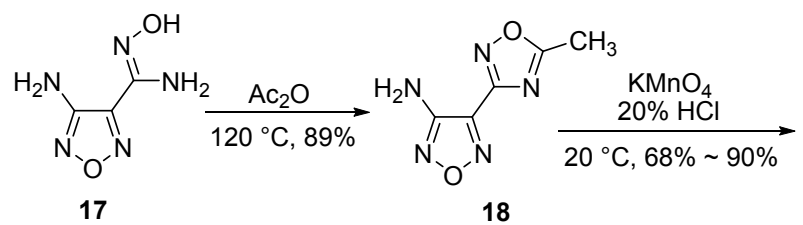<smiles>Cc1nc(-c2nonc2/N=N\c2nonc2-c2noc(C)n2)no1</smiles><smiles>Nc1nonc1/C(=N\O)O[Na]</smiles>

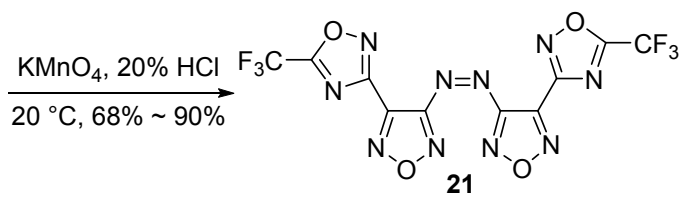

图 15 化合物 19 和 21 合成

Figure 15 Synthesis of compounds 19 and 21

偶氮产物 $(19,21)$. 偶氮结构可有效提高化合物的氮含 量和生成焓, 并保持原有的平面结构 ${ }^{[49-51]}$. 其中, 偶氮 三氟甲基产物(21)密度达 $1.94 \mathrm{~g} \cdot \mathrm{cm}^{-3}$, 理论爆速为 6602 $\mathrm{m} \cdot \mathrm{s}^{-1}$, 撞击感度大于 $80 \mathrm{~J}$, 摩擦感度大于 $360 \mathrm{~N}$.

1.1 .4 偕胺肟与酰胺合成 $1,2,4-$ 嚊二唑

酰胺的反应活性低, 高温下易分解 ${ }^{[52]}$, 因此偕胺肟
与酰胺的反应研究不多. Shaposhnikov 等 ${ }^{[52]}$ 报道了 4-氨 基-1,2,5-呋咱-3-偕胺肜与酰胺的 “一锅” 法合成 1,2,4啞二唑, 收率为 $33 \% \sim 50 \%$ (Eq. 15).

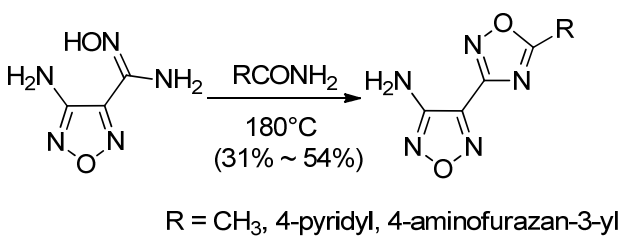

Yang 等 ${ }^{[24]}$ 采用这一方法合成 4-氨基-3-(5-甲基1,2,4-噁二唑-3-基)呋咱(22), 随后利用呋咱上的活性氨 基进行衍生反应：(1)经硝化得到硝氨基(25), 进而利用 酸性氢成盐(26a～26j); (2)与三硝基乙醇反应，经脱水 缩合引入三硝基乙基高能高氧基团(23). 这一系列化合 物密度 $1.63 \sim 1.78 \mathrm{~g} \bullet \mathrm{cm}^{-3}$, 其中化合物 3- $(N, 2,2,2$-四硝 基乙氨基)-4-(5-甲基-1,2,4-噁二唑)呋咱(24)爆速达 8602 $\mathrm{m} \cdot \mathrm{s}^{-1}$, 密度 $1.78 \mathrm{~g} \cdot \mathrm{cm}^{-3}$ (图 16).

\subsection{5 偕胺肜与原甲酸酯合成 $1,2,4$-噁二唑}

Andrianov 等 ${ }^{[53]}$ 发现在醚合三氟化嗍的催化作用下， 原甲酸乙酯与二氨基乙二肜单边的偕胺肜反应，生成 1,2,4-噁二唑-3-偕胺肟(27), 27 再与亚硝酸钠和盐酸反应 得到 1,2,4-噁二唑-3-氯肜(28) (图 17).

Cheng 等 ${ }^{[54]}$ 以 1,2,4-噁二唑-3-氯肟(28)为起始反应 物进行了后续研究，通过硝化、叠氮和与环化等反应， 成功合成了 3-二硝基甲基-1,2,4-噁二唑(31)、5-(1,2,4-啞 二唑-3-基)-1-羟基四唑(33)(及其三种含能盐)和 34 (图 18). 其中，3-二硝基甲基-1,2,4-噁二唑的钾盐(30a)理论

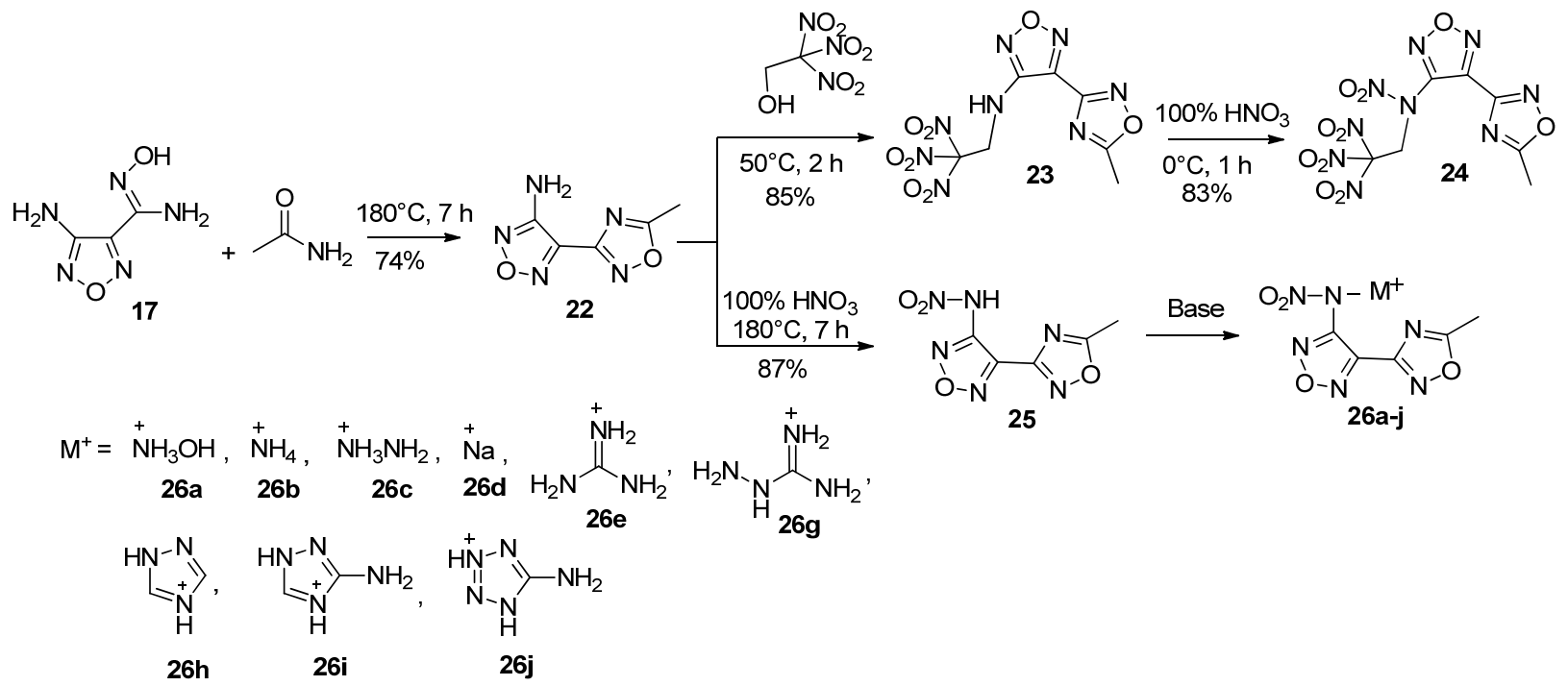

图 16 化合物 27 及其含能离子盐的合成

Figure 16 Synthesis of compound 27 and its energetic ion salts 


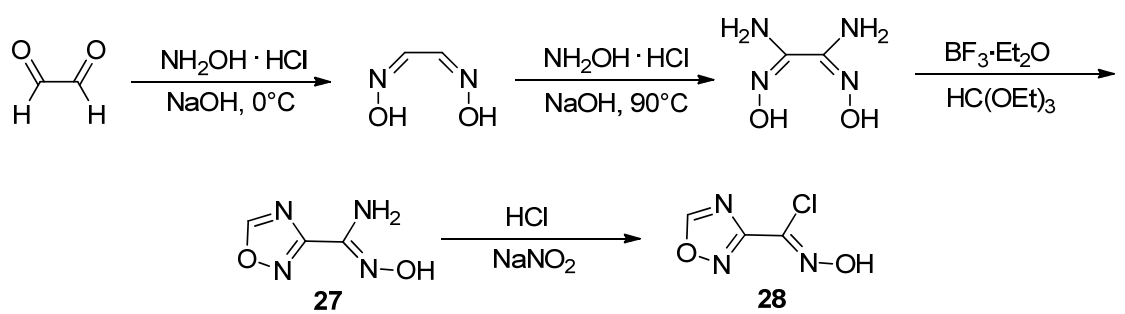

图 17 化合物 $\mathbf{2 8}$ 合成

Figure 17 Synthesis of compound $\mathbf{2 8}$

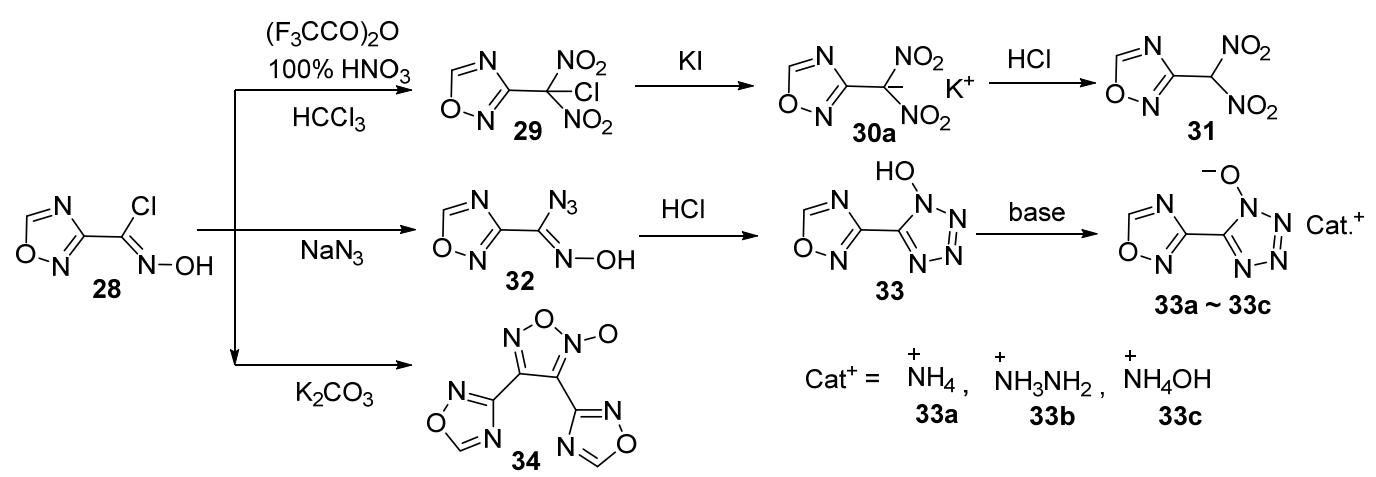

图 18 化合物 33,34 及其含能离子盐的合成

Figure 18 Synthesis of compounds 33, 34 and its energetic ion salts

密度达 $2.07 \mathrm{~g} \bullet \mathrm{cm}^{-3}$, 理论爆速 $8800 \mathrm{~m} \bullet \mathrm{s}^{-1}$. 结果表明 1,2,4-噁二唑-3-氯肜作为重要的含能中间体, 在合成 1,2,4-惡二唑含能衍生物中具有广阔的应用前景.

$\mathrm{Qu}$ 等 ${ }^{[20]}$ 以 4-氨基-3-肟基-1,2,4-噁二唑和原甲酸酯 为原料, 经环化、氧化偶联得到偶氮产物(36) (图 19).

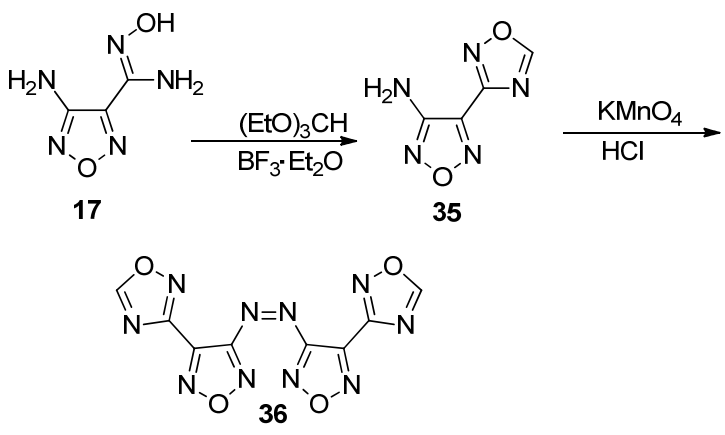

图 19 化合物 $\mathbf{3 5}$ 和 $\mathbf{3 6}$ 合成

Figure 19 Synthesis of compounds $\mathbf{3 5}$ and $\mathbf{3 6}$

\section{2 偕胺肜与腈合成 1,2,4-噁二唑}

\subsection{1 溴化氰与偕胺肜合成 $1,2,4$-噁二唑}

Dimsdale ${ }^{[55]}$ 确定，2,6-二氯苄基偕胺肟与溴化氰在 碳酸氢钾的催化下, 首先发生 $O$-酰化反应而非 $N$-酰化 反应, 随后脱水环化得到 1,2,4-噁二唑(Eq. 16). 可能的 反应机理见图 20 .

1,2,4-噁二唑的 5 位活性氨基可进一步进行硝化、 氧化等反应得到多种结构的含能化合物. Shreeve 等 ${ }^{[56]}$
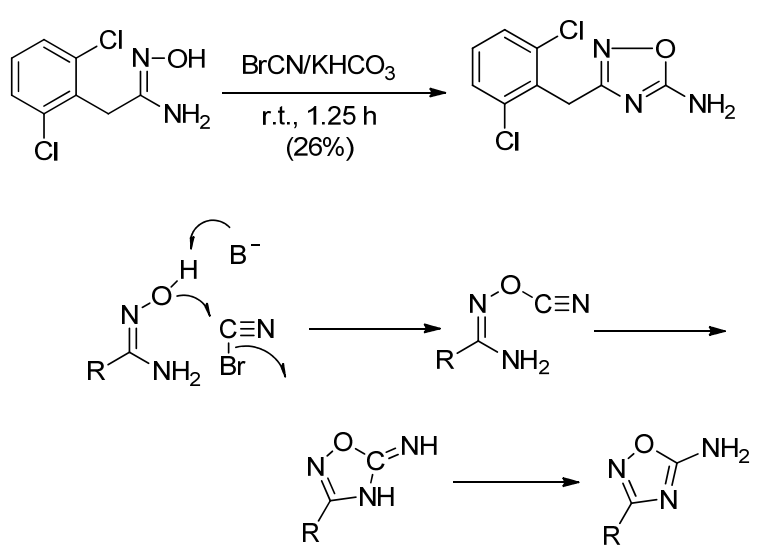

图 20 偕胺肜与溴化氰缩合环化反应机理

Figure 20 Proposed reaction mechanism of amidoximes and cyanogen bromide

以化合物 37 为原料与 $\mathrm{BrCN}$ 合环, 没有得到预期产物 41, 反而获得了两种产物 38 和 39. 这是一种肟基 $\alpha-C$ 发 生 $\mathrm{C}-\mathrm{C}$ 键断裂一偶联构建联 1,2,4-噁二唑的新反应. 将 化合物 38 分离, 再经硝化、成盐等得到 3 种含能离子盐. 其中, 乙胺盐(40b)的 $\mathrm{IS}=25 \mathrm{~J}, \mathrm{FS}=40 \mathrm{~J}$, 理论爆速 9078 $\mathrm{m} \cdot \mathrm{s}^{-1}$, 是一种新型高能钝感含能离子盐(图 21).

Shaposhnikov 等 ${ }^{[57]}$ 首次报道了 3-氨基-4-(5-氨基1,2,4-噁二唑)呋咱(42)的合成. Shreeve 等 ${ }^{[58]}$ 以 42 为原料, 再经过硝化、成盐等反应得到其含能离子盐. 其中 $42 \mathrm{c}$ 密度为 $1.85 \mathrm{~g} \bullet \mathrm{cm}^{-3}$, 理论爆速达 $9046 \mathrm{~m} \bullet \mathrm{s}^{-1}$, 撞击感 


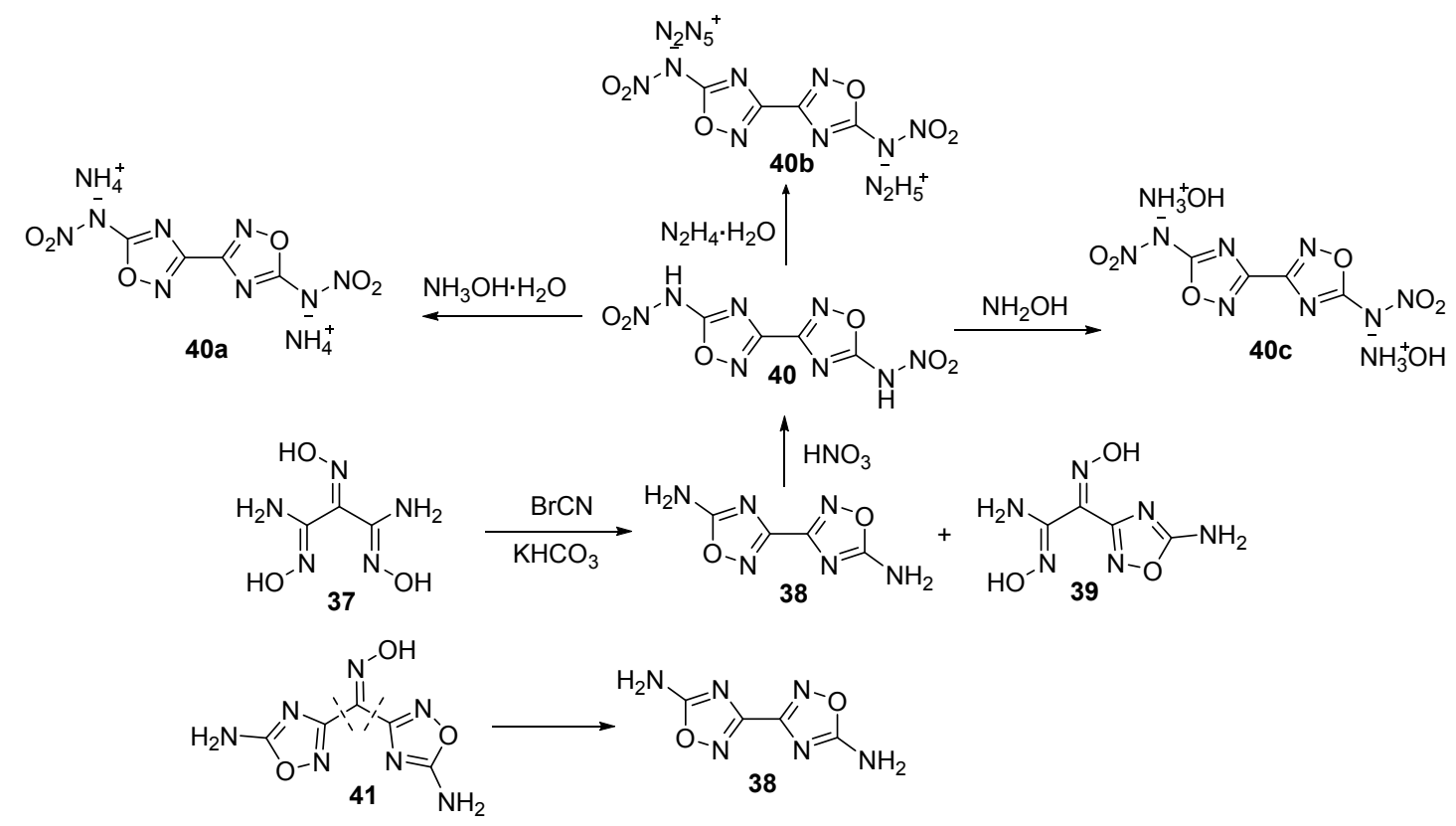

图 21 化合物 $\mathbf{3 8 ,}, \mathbf{3 9}$ 及其含能离子盐的合成

Figure 21 Synthesis of compounds 38, 39 and its energetic ion salts

度为 $16 \mathrm{~J}$, 摩擦感度为 $160 \mathrm{~N}$ (图 22).

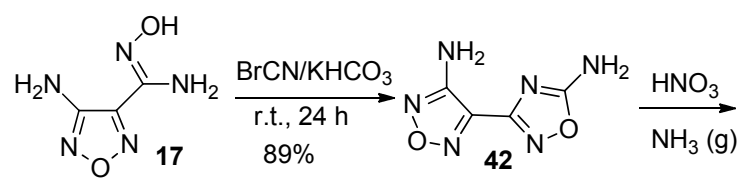<smiles></smiles>

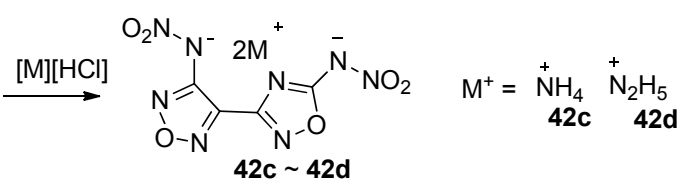

图 22 含能离子盐 $42 a \sim 42 d$ 的合成

Figure 22 Synthesis of the energetic ion salts $42 a \sim 42 d$

$\mathrm{Qu}$ 等 ${ }^{[20]}$ 以 42 为原料, 经氧化偶联得到 43 (由于呋 咱侧链的氨基活性高于异呋咱侧链的氨基, 偶氮反应优 先在呋咱侧链氨基发生), 随后硝化得到 DDAzF (44). 其中, 44 密度高达 $2.12 \mathrm{~g} \bullet \mathrm{cm}^{-3}$, 理论爆速达 $10114 \mathrm{~m}$ • $\mathrm{s}^{-1}$, 高于 RDX 和 HMX, 与 CL-20 相当, 分解温度为 $317{ }^{\circ} \mathrm{C}$, IS 和 FS 均在可接受范围内, 是一个综合性能突 出的高能材料, 其高密度可能归因于硝氨基参与多个分 子氢键相互作用和其共轭平面结构(未得到单晶结构) (图 23).

$\mathrm{Lu}$ 等 ${ }^{[59]}$ 则利用 42 的两个氨基的活性不同, 先将呋 咱侧链的氨基氧化成硝基, 得到化合物 45 , 再用

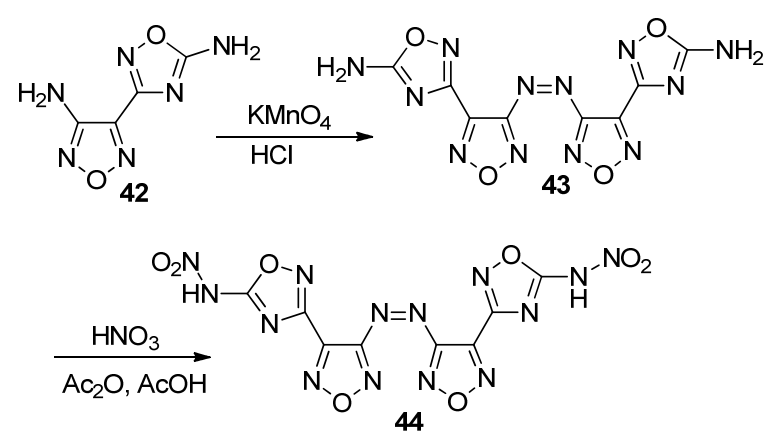

图 23 化合物 43 和 44 合成

Figure 23 Synthesis of compounds 43 and 44

$\mathrm{KMnO}_{4} / \mathrm{HCl}$ 处理, 得到偶氮化合物 46, 总收率约为 $40.33 \%$. 该化合物的理论密度达 $1.92 \mathrm{~g} \cdot \mathrm{cm}^{-3}$, 理论爆速 达 $9240 \mathrm{~m} \bullet \mathrm{s}^{-1}$, IS 为 $18 \mathrm{~J}, \mathrm{FS}$ 为 $220 \mathrm{~N}$, 熔点 $157{ }^{\circ} \mathrm{C}$, 分 解点 $256{ }^{\circ} \mathrm{C}$, 综合性能优于 HMX, 是一种有着潜在应 用前景的高能针感炸药(图 24).

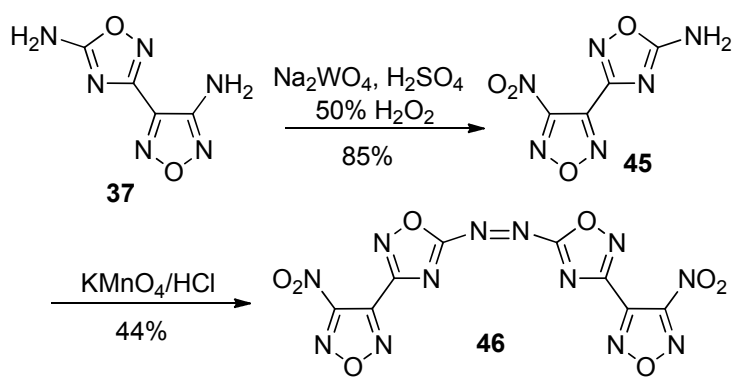

图 24 化合物 46 合成

Figure 24 Synthesis of compound $\mathbf{4 6}$ 


\subsection{2 有机腈与偕胺肜合成 $1,2,4$-噁二唑}

腈和偕胺肟的反应分两种反应机理进行: (i) $O$-酰化 合环反应; (ii)腈氧化物的 1,3-偶极环加成.

Yarovenko 等 ${ }^{[60]}$ 首次报道了有机腈与偕胺肜合成 1,2,4-啞二唑. 乙腈与苯甲酰肜反应需要在密封管中进 行, $180{ }^{\circ} \mathrm{C}$ 反应 $12 \mathrm{~h}$, 产率仅为 $21 \%$ (Eq. 17). 该反应可 能的机理为 $O$-酰化环化, 见图 25 .

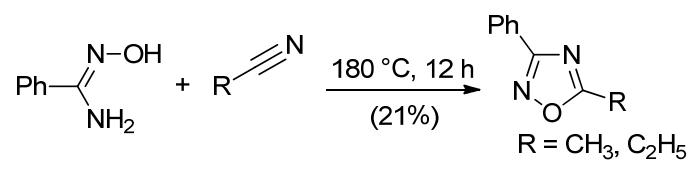

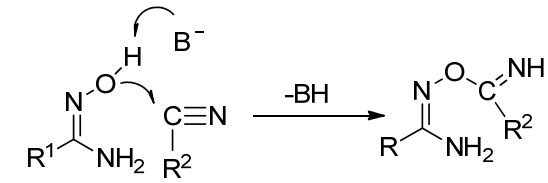

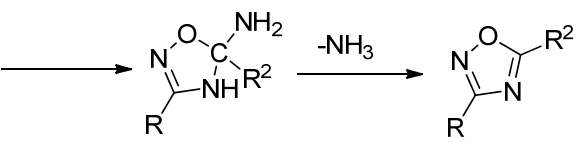

图 25 偕胺肜与有机腈合环反应机理

Figure 25 Proposed reaction mechanism of amidoxime and organic nitrile

另一种反应机理为 $[3+2]$ 偶极环加成, 这种反应方 式可大大提升产率. Augustine 等 ${ }^{[61]}$ 利用 PTSA- $\mathrm{ZnCl}_{2}$ 作 催化剂, 偕胺肜与有机腈以高收率(77\% 91\%)得到 25 种 1,2,4-噁二唑(Eq. 18). 可能的原因是 PTSA- $\mathrm{ZnCl}_{2}$ 活 化偕胺肟离去一分子 $\mathrm{NH}_{3}$ 形成氧化腈, 与腈发生 $[3+2]$ 偶极环加成反应(图 26).

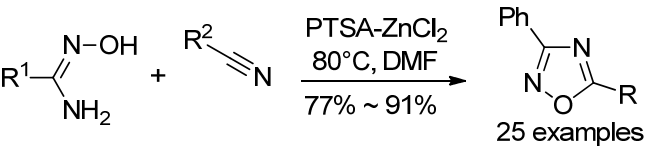

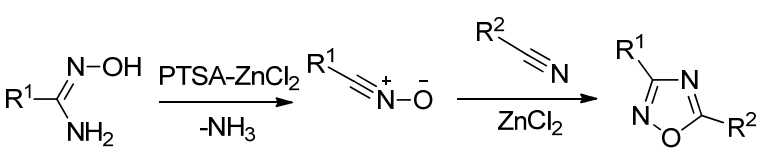

\section{图 26 偕胺肜与有机腈合环反应机理}

Figure 26 Proposed reaction mechanism of amidoxime and organic nitrile via PTSA- $\mathrm{ZnCl}_{2}$

Shreeve 等 ${ }^{[58]}$ 利用 17 与 3-氨基-4-氰基呋咱(47), 在 $\mathrm{TsOH} / \mathrm{ZnCl}_{2}$ 催化体系中环化得到双呋咱基 1,2,4-噁二唑 化合物 48, 经硝化、成盐反应得到硝氨基化合物 49 及 其含能离子盐 $(49 \mathrm{a} \sim 79 \mathrm{~b})$, 其中 $49 \mathrm{a}$ 的密度为 $1.71 \mathrm{~g} \cdot$ $\mathrm{cm}^{-3}$, 爆速 $8603 \mathrm{~m} \cdot \mathrm{s}^{-1}$ (图 27).

\section{$1.3 N$-氧基亚胺与羟胺合成 3 -氨基-1,2,4-噁二唑}

1963 年, Huffman 等 ${ }^{[62]}$ 首次报道了利用 $N$-氰基亚胺
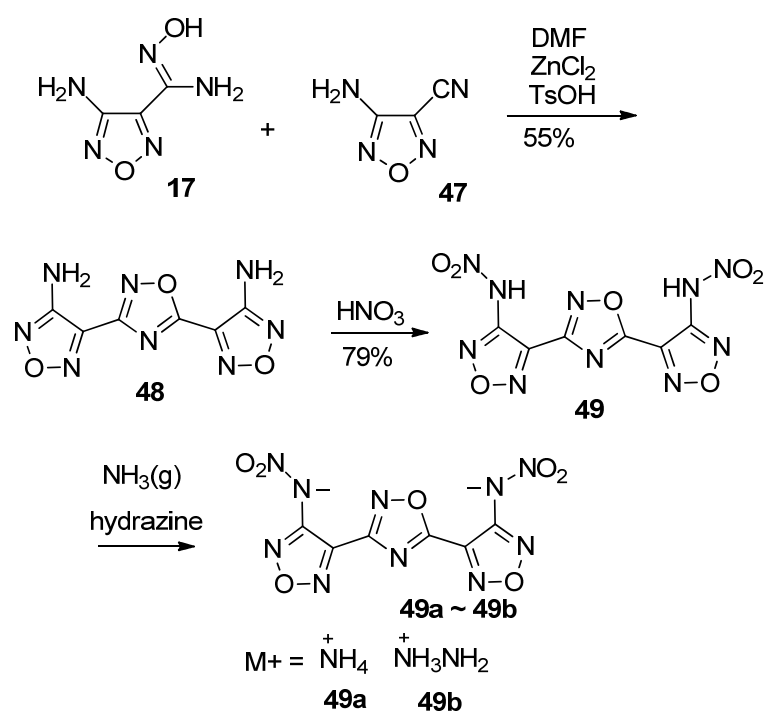

图 27 化合物 48,49 及其含能离子盐的合成

Figure 27 Synthesis of compounds 48, 49 and its energetic ion salts

和羟胺盐酸盐合成 3-氨基-1,2,4-噁二唑(Eq. 19).

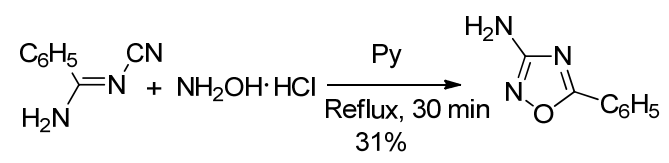

Dimsdale 等 ${ }^{[55]}$ 分析了该反应可能的反应机理，反应 可能通过两种方式进行: (i)差胺氮首先亲核加成氰基碳, 随后羟胺氧进攻亚氨酸酯的碳环化后脱 HX; (ii)羟胺氧 首先亲核加成亚氨酸酯的碳氰基碳，随后羟胺氮进攻氰 基碳后脱 HX 环化(图 28).

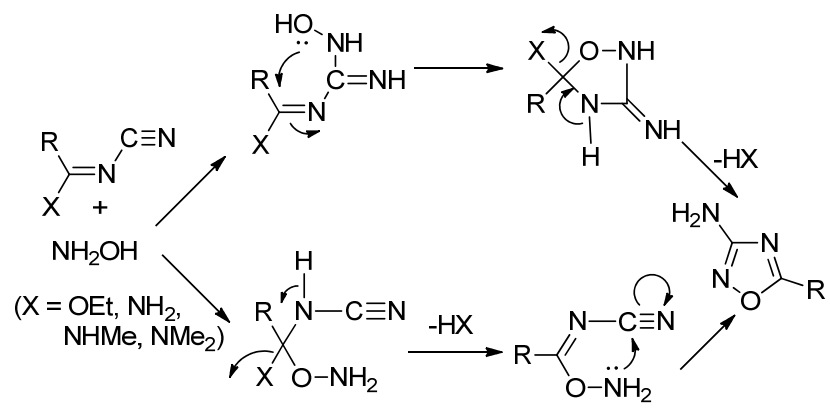

图 $28 N$-氰基亚胺与羟胺环化反应机理

Figure 28 Proposed cyclization reaction mechanism of $\mathrm{N}$-cyanoimine and hydroxylamine

1.3.1 1,3-二氨基-1,2,4-噁二唑及其含能衍生物合成

Huttunen 等 ${ }^{[63]}$ 以二氧基胺钠为原料，在二甲基胺的 催化作用下与盐酸羟胺反应得到重要的反应前体化合 物 1,3-二氨基-1,2,4-噁二唑(50). 可能的机理是: 二氧基 胺钠与二甲基胺发生单边亲核加成反应，再与盐酸羟胺 发生加成，碱性条件下 $\mathrm{O}$ 进攻 $\mathrm{C}$ 发生合环反应，再脱去 
一分子二甲基胺得到 51(图 29).

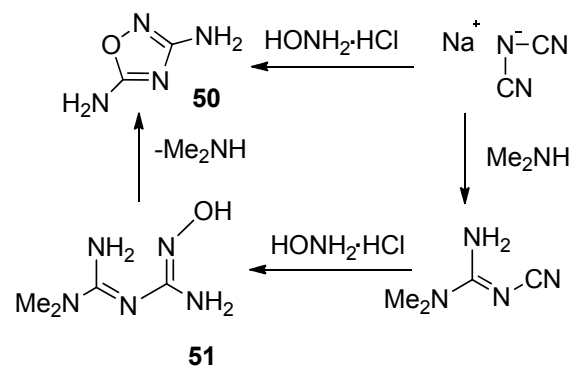

图 29 化合物 $\mathbf{5 0}$ 的合成

Figure 29 Synthesis of compound $\mathbf{5 0}$

这一反应中间体 $\mathbf{5 0}$ 在 3,5 位均有氨基, 利用不同位 点氨基的反应活性不同, 通过控制反应条件, 可得到多 种性能优异的含能化合物。

Shreeve 课题组 ${ }^{[64]}$ 以 $\mathbf{5 0}$ 为原料, 经高锰酸钾/盐酸体 系氧化，1,2,4-噁二唑的 3 位氨基可偶联生成偶氮产物 48. 然后, 进一步采用不同的硝化体系得到不同的硝化 产物 ${ }^{[65]}$. 偶氮产物在 $100 \% \mathrm{HNO}_{3}$ 中硝化, 可得到 5,5'二硝氨基偶氮-1,2,4-留二唑 52, 进一步合成相应的含能 离子盐. 这一系列化合物爆速较高(8381 9243 $\mathrm{m} \bullet \mathrm{s}^{-1}$ ), 其中羟胺含能离子盐 $(49 \mathrm{a})$ 密度为 $1.864 \mathrm{~g} \cdot \mathrm{cm}^{-3}$, 爆速 $9243 \mathrm{~m} \cdot \mathrm{s}^{-1}, \mathrm{FS}$ 为 $160 \mathrm{~N}$, IS 为 $10 \mathrm{~J}$, 是一种具有潜在应 用价值的高能离子盐; 偶氮产物在硝酸/醋䣶体系中硝
化，可得到偶氮 1,2,4-噁二唑酮化合物 $\mathbf{5 0}$, 中和得到 3 种盐衍生物 $(\mathbf{5 0 a} \sim 50 \mathrm{c})^{[64]}$ (图 30).

如采用 $\mathrm{Na}_{2} \mathrm{WO}_{4} / \mathrm{H}_{2} \mathrm{O}_{2} / \mathrm{H}_{2} \mathrm{SO}_{4}$ 体系氧化 $\mathbf{5 0}$, 可以得 到 3-位硝化的产物 3-硝基-5-氨基-1,2,4-噁二唑(55). Chen 等 ${ }^{[66]}$ 在二氯异氧尿酸钠 $(\mathrm{SDCI})$ /醋酸条件下处理 55 , 同时得到以一个氮相连的二硝基噁二唑的钠盐(56) 和三氮烯相连的二硝基噁二唑的钠盐(57), 并获得了其 它 6 种含能盐. 其中, 双(3-硝基-1,2,4-惡二唑-5-基)酰胺 胍盐密度为 $1.799 \mathrm{~g} \cdot \mathrm{cm}^{-3}$, 爆速 $8351 \mathrm{~m} \cdot \mathrm{s}^{-1}$, 分解温度 ( $\left.T_{\mathrm{dec}}\right) 241{ }^{\circ} \mathrm{C}, \mathrm{IS}>40 \mathrm{~J}$ (图 31).

Shreeve 等 ${ }^{[67]}$ 以 $\mathbf{5 0}$ 为原料, 在醚合三氟化嗍的催化 下，氯甲酸乙酯仅与一个氨基发生酰胺化反应，硝化后 碱性条件水解得到 $58 \mathrm{a}$ 及其 2 种富氮盐 $(58 b \sim 58 c)$. 这 一系列化合物爆速较高 $\left(8493 \sim 8897 \mathrm{~m} \cdot \mathrm{s}^{-1}\right)$, 其中 $\mathbf{5 8 a}$ 的 爆速 $8897 \mathrm{~m} \cdot \mathrm{s}^{-1}, \mathrm{IS}=20 \mathrm{~J}, \mathrm{FS}=240 \mathrm{~N}$, 且原材料低廉, 步骤少, 综合性能与 RDX 相当, 是一种潜在的 RDX 替 代物(图 32).

Chen 等 ${ }^{[68]}$ 在 $\mathrm{P}_{2} \mathrm{O}_{5} / \mathrm{HNO}_{3}$ 条件下对 $\mathbf{5 5}$ 进行硝化后得 到硝氨化产物 (60), 碱性条件水解得到五种含能盐 $(60 \mathrm{a} \sim 60 \mathrm{e})$. 其中 $60 \mathrm{a}$ 的理论密度 $1.888 \mathrm{~g} \cdot \mathrm{cm}^{-3}$, IS $>40$ $\mathrm{J}$, 理论爆速 $9354 \mathrm{~m} \cdot \mathrm{s}^{-1}$, 熔点 $146 \sim 148{ }^{\circ} \mathrm{C}$, 分解温度 $258{ }^{\circ} \mathrm{C}$. 是一种综合性能优于 $\mathrm{RDX}$ 的含能离子盐(图 $33)$.

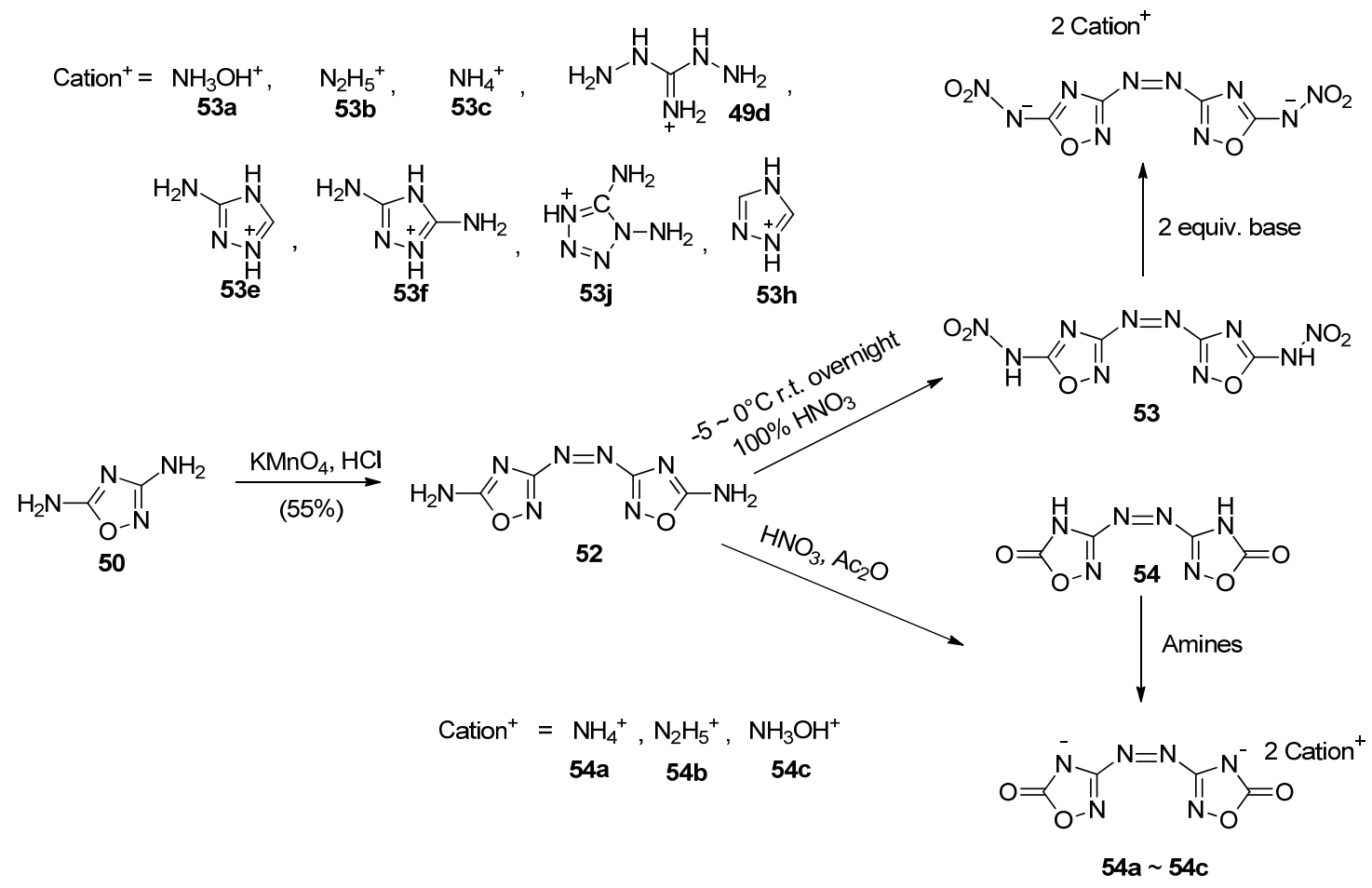

图 30 化合物 $\mathbf{5 3}$ 和 $\mathbf{5 4}$ 及其含能离子盐的合成

Figure 30 Synthesis of compounds $\mathbf{5 3}$ and $\mathbf{5 4}$ and its energetic ion salts 


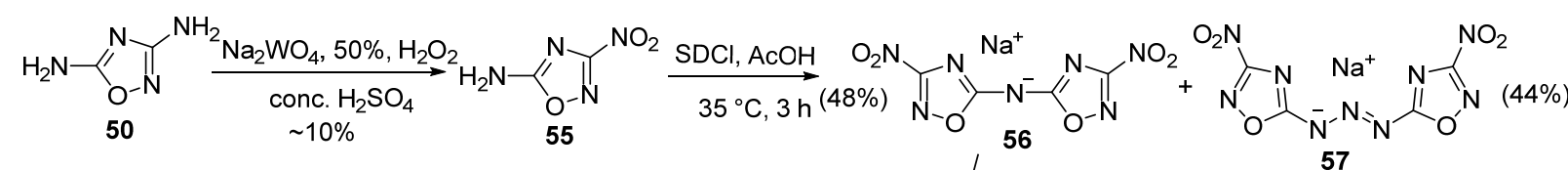<smiles>[N]=C(N)[15NH2]</smiles><smiles></smiles>
1) $\mathrm{AgNO}_{3}, \mathrm{H}_{2} \mathrm{O}$ 2) $\mathrm{M} \cdot \mathrm{HCl}$

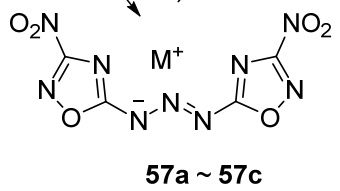

图 31 化合物 56 和 57 及其含能离子盐的合成

Figure 31 Synthesis of compounds $\mathbf{5 6}$ and $\mathbf{5 7}$ and its energetic ion salts<smiles>CCOC(=O)COC(=O)Nc1nc(N)no1</smiles>

50

58<smiles>Nc1no/c(=N\[N+](=O)[O-])[nH]1</smiles>

图 32 化合物 59 及含能离子盐

Figure 32 Synthesis of compound $\mathbf{5 9}$ and its energetic ion salts<smiles>Nc1nc([N+](=O)[O-])no1</smiles><smiles>CC(C)(O)O[N+](=O)[O-]</smiles>

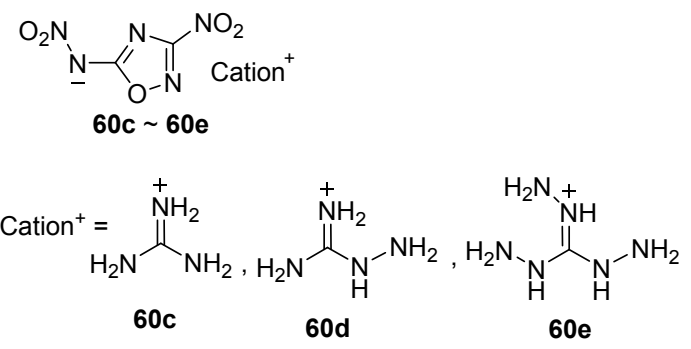

图 33 化合物 $\mathbf{5 7}$ 及其含能离子盐的合成

Figure 33 Synthesis of compound $\mathbf{5 7}$ and its energetic ion salts

此外, Chen 等 ${ }^{[69]}$ 以化合物 61 为原料, 在 $\mathrm{Cs}_{2} \mathrm{CO}_{3}$ 催 化下, 55 成功将四嗪上的氮杂五元环取代, 得到化合物
62, 经 $\mathrm{P}_{2} \mathrm{O}_{5} / \mathrm{HNO}_{3}$ 硝化得到化合物 63 和化合物 $\operatorname{TTNOA}(64), 63$ 的密度为 $1.84 \mathrm{~g} \cdot \mathrm{cm}^{-3}(298 \mathrm{~K})$, 理论爆速 $8976 \mathrm{~m} \cdot \mathrm{s}^{-1}, \mathrm{IS}>40 \mathrm{~J}$, 综合性能优良(图 34).<smiles>[CH2+][NH2+]c1cc(C)n(-c2nnc3nnc(N)n3n2)n1</smiles>

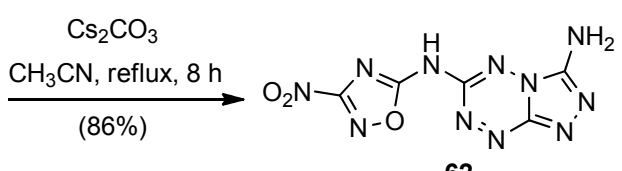<smiles>[R6]O[N+](=O)[O-]</smiles><smiles>O=[N+]([O-])c1noc(Nc2nnc3nnc([N+](=O)[O-])n3n2)n1</smiles>

图 34 化合物 63 和 64 的合成

Figure 34 Synthesis of compounds 63 and 64

\section{4 二氨基甘脲重排合成 1,2,4-噁二唑}

二氨基甘艮等氮杂环的重排也可得到 1,2,4-噁二唑. Chen 等 ${ }^{[17]}$ 在接近中性条件下用二甲基过氧化酮处理二 氨基甘嫝，重排后得到 3-硝基-5-胍基-1,2,4-噁二唑(66). 利用胍基良好的反应活性，采用不同的硝化反应条件的 到 55, 60. 其中, 60 密度为 $1.883 \mathrm{~g} \bullet \mathrm{cm}^{-3}$, 爆速 $9095 \mathrm{~m} \bullet$ $\mathrm{s}^{-1}$, 分解温度 $\left(T_{\mathrm{dec}}\right) 219{ }^{\circ} \mathrm{C}$, IS 为 $15 \mathrm{~J}$ (图 35).

\subsection{1,2,4-噁二唑含能化合物性能研究}

将 1,2,4-噁二唑结构单元引入中含能化合物中, 期 望利用 1,2,4-噁二唑的稳定性和不敏感性，同时提高化 合物的具备高氧平衡值, 设计、合成新型的高能低感含 能材料. 


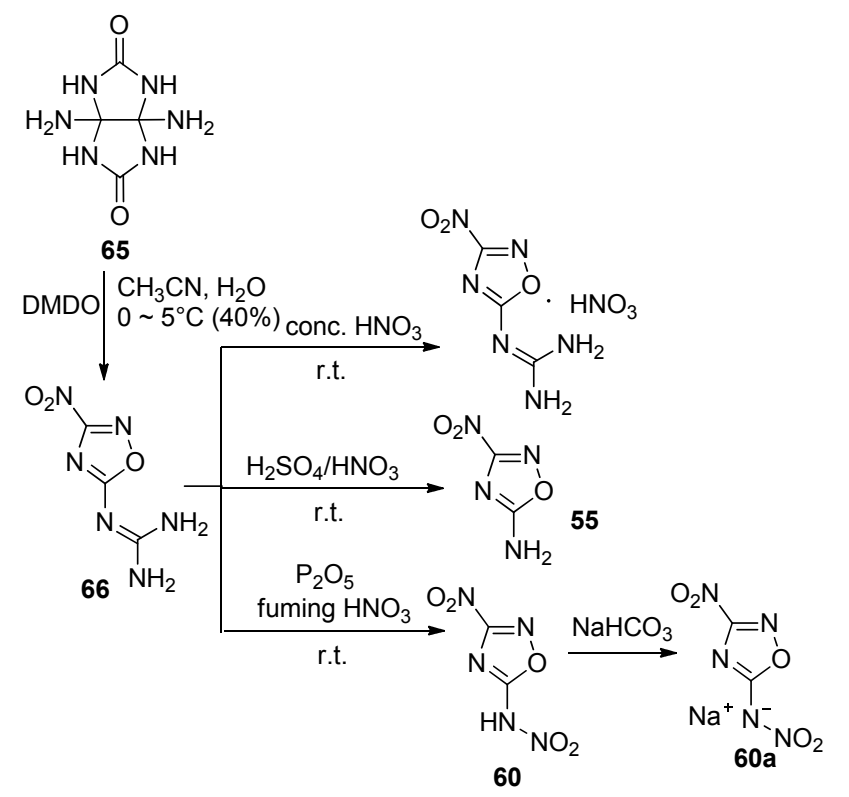

图 35 化合物 60 及含能离子盐的合成

Figure 35 Synthesis of compound 60 and its energetic ion salts

1,2,4-䜿二唑基含能化合物性能对比可以看出, 此 类化合物普遍具有感度低, 热稳定性好的特点; 共轭型 化合物(如偶氮类)具有更好的综合性能. 其中不乏一些 性能优异的化合物, 如 DDAzF 的密度高达 $2.12 \mathrm{~g} \cdot$ $\mathrm{cm}^{-3}$, 理论爆速达 $10114 \mathrm{~m} \cdot \mathrm{s}^{-1}$, 高于 RDX 和 HMX, 与 CL-20 相当, 分解温度为 $317{ }^{\circ} \mathrm{C}, \mathrm{IS}$ 和 FS 均在可接受范 围内, 是一个综合性能突出的新型含能材料, 其高密度 可能归因于硝氨基参与多个分子氢键相互作用和其共 轭平面结构.

表 1 1,2,4-惡二唑含能化合物与 RDX 及 HMX 性能对比

Table 1 Properties of some 1,2,4-oxadiazole energetic compounds compared with RDX and HMX

\begin{tabular}{lcccccc}
\hline \multicolumn{1}{c}{ Substrate } & $\mathrm{FS} / \mathrm{N}$ & $\mathrm{IS} / \mathrm{J}$ & $T_{\mathrm{dec}}{ }^{\circ} \mathrm{C}$ & $\begin{array}{c}p / \\
\left(\mathrm{g} \bullet \mathrm{cm}^{-3}\right)\end{array}$ & $\begin{array}{c}V_{\mathrm{det}} \\
\left(\mathrm{m} \bullet \mathrm{s}^{-1}\right)\end{array}$ & $\begin{array}{c}H_{50} / \\
\mathrm{cm}\end{array}$ \\
\hline $\mathrm{RDX}^{[70]}$ & 120 & 7.4 & 210 & 1.86 & 8830 & 22 \\
$\mathrm{HMX}^{[71]}$ & 120 & 7.0 & 260 & 1.91 & 9110 & - \\
$\mathrm{DDAzF}^{[20]}$ & 80 & 6.0 & 317 & 2.12 & 10114 & - \\
$\mathrm{LLM}-200^{[46]}$ & - & - & 295 & 1.94 & 8780 & 62 \\
$\mathbf{2}^{[7]}$ & 160 & 4.0 & 156 & 1.95 & 8935 & - \\
$\mathbf{3}^{[38]}$ & 192 & 10 & 124 & 2.04 & 8367 & - \\
$\mathbf{4}^{[39]}$ & 80 & 10 & 151 & 2.02 & 8814 & - \\
$\mathbf{4 2}^{[59]}$ & 220 & 18 & 256 & 1.92 & 9240 & - \\
$\mathbf{5 7 a}^{[68]}$ & - & $>40$ & 258 & 1.89 & 9319 & - \\
$\mathbf{6 0}^{[17]}$ & - & 15 & 219 & 1.88 & 9095 & - \\
\hline
\end{tabular}

\section{1,3,4-噁二唑含能化合物的合成及性能}

\section{1 酰肼与溴化氭合成 1,3,4-噁二唑}

酰肼和溴化氧的反应活性高. 在弱碱催化下, 即可 以高收率获得环化产物. 可能的反应机理见图 36.

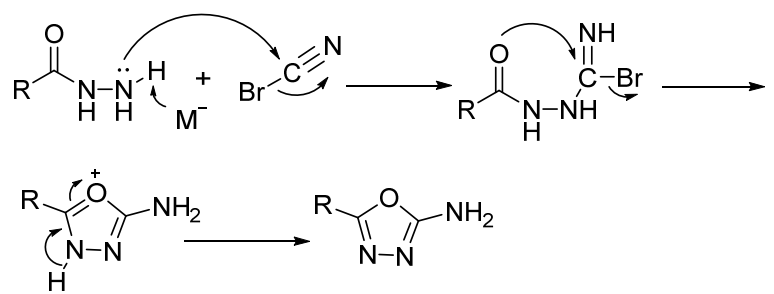

图 36 酰肼与溴化氧环化反应机理

Figure 36 Proposed cyclization reaction mechanism of acylhydrazide with cyanogen bromide

Klapötke 等 ${ }^{[72]}$ 利用二草酰肼与溴化氰缩合得到反 应前体 5,5'-二氨基-1,3,4-噁二唑(62), 然后用 $100 \%$ $\mathrm{HNO}_{3}$ 硝化得到化合物 63, 进一步成盐得到 6 种含能离 子盐. 其中铵盐(63a)的理论密度达 $1.95 \mathrm{~g} \cdot \mathrm{cm}^{-3}$, 理论爆 速达 $9255 \mathrm{~m} \cdot \mathrm{s}^{-1}, \mathrm{IS}=10 \mathrm{~J}, \mathrm{FS}=360 \mathrm{~N}$, 综合性能优于 $\mathrm{RDX}$, 且反应步骤短, 收率高, 是一种有潜在应用价值 的钝感高能离子盐. Zhang 等 ${ }^{[73]}$ 对化合物 63 进行了进一 步的研究, 成功获得了单晶结构, 并进行了系统解析, 其晶体密度达 $1.99 \mathrm{~g} \cdot \mathrm{cm}^{-3}(298 \mathrm{~K})$, 实测爆速 $9481 \mathrm{~m} \cdot$ $\mathrm{s}^{-1}, \mathrm{IS}=5 \mathrm{~J}, \mathrm{FS}=60 \mathrm{~N}$, 感度略低于 $\varepsilon-C L-20$, 反应路径 短, 原料低廉, 是一种具有应用潜力的、爆轰性能突出 的新型高能炸药(图 37).
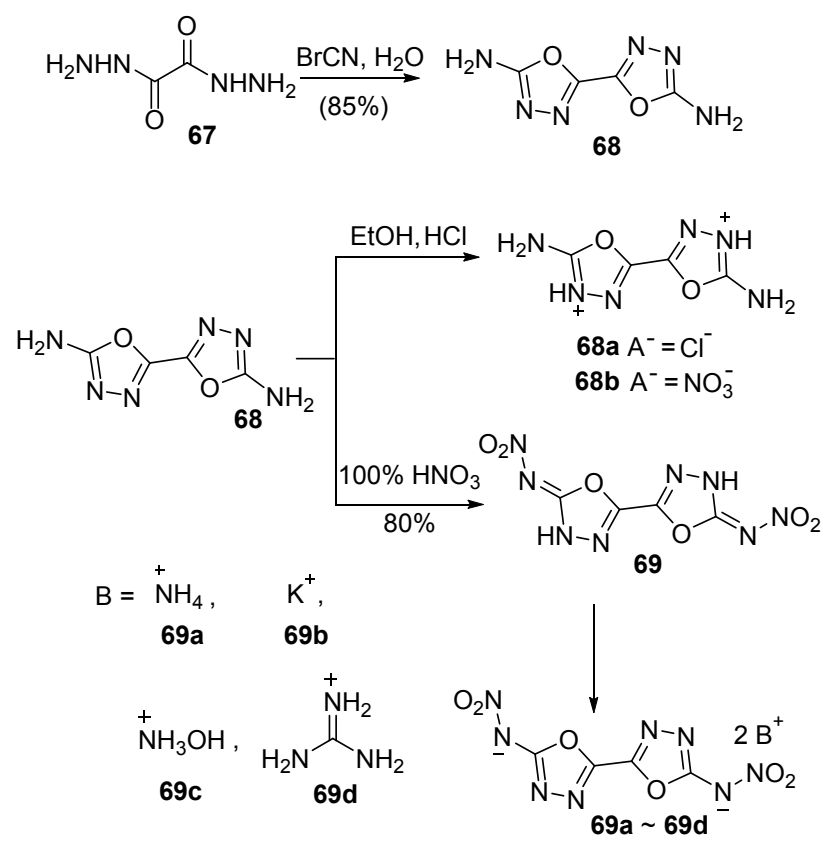

图 37 化合物 68 和 69 及含能离子盐的合成

Figure 37 Synthesis of compounds 68 and 69 and its energetic ion salts

Shreeve 等 ${ }^{[74]}$ 利用澳化氧与 4-氨基-1,2,5-惡二唑-3乙酰肼缩合得到 3-氨基-4-(2-氨基-1,3,4-噁二唑 基)-1,2,5-噁二唑(71). 由于与 1,3,4-噁二唑环的氨基非 常不活泼, 经硝化仅能得到单硝化产物, 进一步进行中 
和反应合成系列含能离子盐 $(72 \mathrm{a} \sim 72 \mathbf{i})$, 其理论密度 $1.61 \sim 1.81 \mathrm{~g} \bullet \mathrm{cm}^{-3}$, 理论爆速 $7493 \sim 8711 \mathrm{~m} \bullet \mathrm{s}^{-1}$ (图 38).

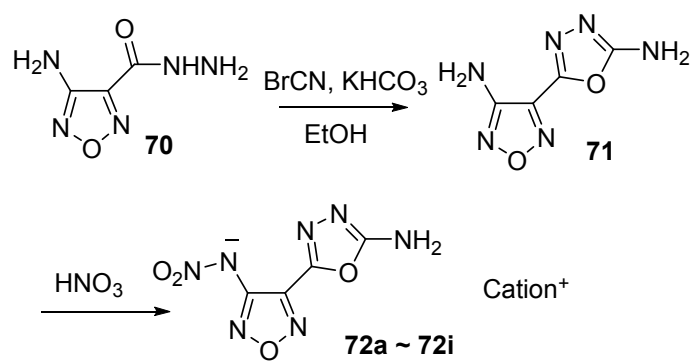

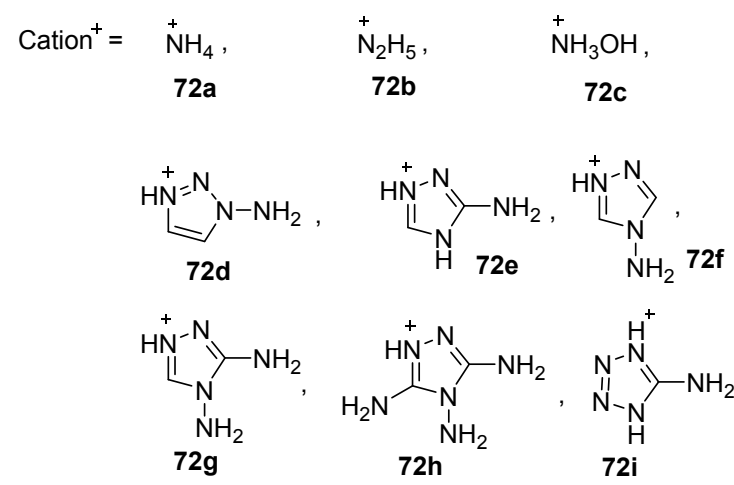

图 38 含能离子盐 $72 a \sim 72 i$ 的合成

Figure 38 Synthesis of energetic ion salts $72 \mathbf{a} \sim 72 \mathbf{i}$

\section{2 二酰肼脱水合成 1,3,4-噁二唑}

\section{2 .1 二酰肼的构建}

二酰肼一般是由酰肼和羧酸衍生物的合成, 反应可 在添加缚酸剂的条件下进行, 不同的取代基导致反应收 率的不同. 可能的反应机理如图 39(以吡定为例).<smiles>[R]C(=O)NNCCCCCCCC([R])[X]</smiles><smiles>[R]C([X])([O-])NNC(=O)NCCCC</smiles><smiles>[R]C(=O)NNC([R])([R])CC</smiles><smiles>[R]C(=O)NNC([R])=O</smiles>

图 39 二酰肼合成反应机理

Figure 39 Proposed synthesis reaction mechanism of dihydrazide

酰氯 ${ }^{[75,76]}$ 和酸酐 ${ }^{[77,78]}$ 反应活性高, 在室温下即完成 反应，收率与取代基有关(Eq. 20). 酯的反应活性较低, 通常需要在高温下回流 ${ }^{[79]}$ 才能反应(Eq. 21).
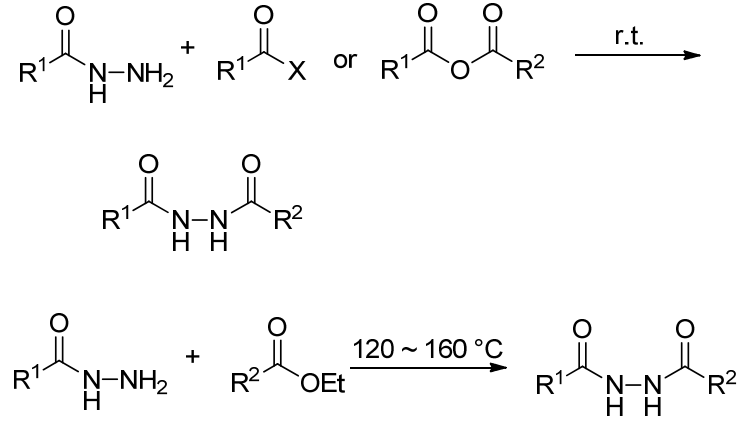

\subsection{2 脱水环化}

通常，二酰肼的脱水环化多使用的是五氧化二

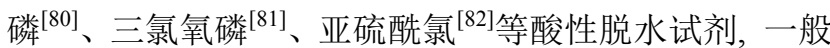
需要在长时间加热条件下完成环化，后处理困难，这种 试剂不利于酸敏感型原料的环化. 目前的研究主要侧重 于改良实验条件，使反应条件更温和，产率更高，副产 物少且易于分离. Ishikawa 等 ${ }^{[83]}$ 在几乎中性的条件下使 用 2-氯-1,3-二甲基咪唑氯化物(DMC)作为强力脱水剂, 在室温下用的 DMC 处理二酰基肼，以优异的收率得到 1,3,4-噁二唑(Eq. 22).

$\underset{\mathrm{HN}-\mathrm{NH}}{\stackrel{\mathrm{Et} \mathrm{N}_{3}, \mathrm{CH}_{2} \mathrm{Cl}_{2} \text {, r.t. }}{(86 \% \sim 100 \%)}}$

James 等 ${ }^{[84]}$ 用二酰肼在六氯乙烷/乙腈 $/ N, N$-二异丙 基乙胺 Hunig 碱的体系中进行处理，三苯基膦作为温和 有效的环化脱水剂迅速进行脱水环化，在室温下即可得 到产物噁二唑(Eq. 23).

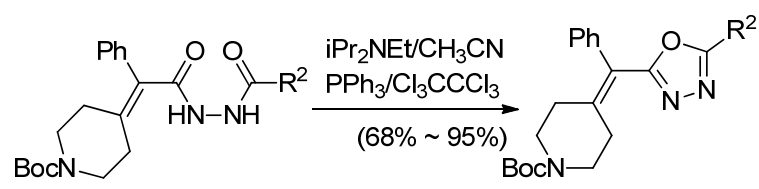

Liras 等 ${ }^{[85]}$ 以二酰基肼为原料，以二氯甲烷作溶剂, 在吡啶/三氟甲磺酸酐体系中, 在温和的温度 $\left(-10{ }^{\circ} \mathrm{C}\right.$ 至环境温度)下即可生成 1,3,4-噁二唑(Eq. 24).

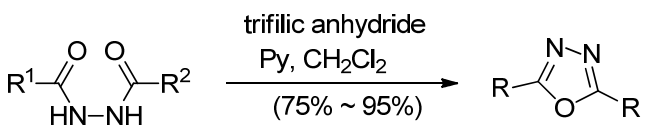

\subsection{3 相关含能衍生化合物的合成}

Sharnin ${ }^{[86]}$ 首次报道了 2,5 -二三硝基苯基-1,3,4-噁二 唑(76)的合成，用三硝基苯酰肼和三硝基苯酰氯反应， 再在 $\mathrm{POCl}_{3}$ 下脱水环化, 得化合物 76 (图 40).

Klapötke 课题组 ${ }^{[21]}$ 三硝基甲苯(77)为原料, 经酰化 得三硝基苯酰氯，与草酰肼反应得到二酰肼，随后在 
<smiles>[Z4]c1cc([N+](=O)[O-])c(C(=O)NN)c([N+](=O)[O-])c1</smiles><smiles>O=C(NNC(=O)c1c([N+](=O)[O-])cc([N+](=O)[O-])cc1[N+](=O)[O-])c1c([N+](=O)[O-])cc([18O])cc1[N+](=O)[O-]</smiles><smiles>Nc1cc([N+](=O)[O-])cc([N+](=O)[O-])c1-c1nnc(-c2c([N+](=O)[O-])cc([N+](=O)[O-])cc2[N+](=O)[O-])o1</smiles>

图 40 化合物 76 及含能离子盐的合成

Figure 40 Synthesis of compound 76 and energetic ion salts

20\%的发烟硫酸下脱水, 环化得到 TKX-55 (79), 其有密 度高 $\left(1.837 \mathrm{~g} \bullet \mathrm{cm}^{-3}\right)$ 、爆轰性能好(爆速 $8030 \mathrm{~m} \bullet \mathrm{s}^{-1}$, 爆压 $27.3 \mathrm{GPa}) 、$ 热稳定性好(DSC 初始分解温度 $335{ }^{\circ} \mathrm{C}$ )、感 度低(FS>360 N)的特点, 综合性能优异(图 41).

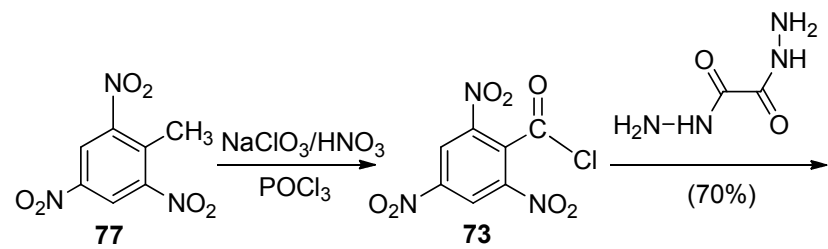<smiles>O=C(NNC(=O)c1c([N+](=O)[O-])cc([N+](=O)[O-])cc1[N+](=O)[O-])C(=O)NNC(=O)c1c([N+](=O)[O-])cc([N+](=O)[O-])cc1[N+](=O)[O-]</smiles><smiles>O=[N+]([O-])c1cc([N+](=O)[O-])c(-c2nnc(-c3nnc(-c4c([N+](=O)[O-])cc([N+](=O)[O-])cc4[N+](=O)[O-])o3)o2)c([N+](=O)[O-])c1</smiles>

图 41 化合物 TKX-55 (79)的合成

Figure 41 Synthesis of compound TKX-55 (79)

\section{3 四唑环重排得 1,3,4-噁二唑}

Klapötke 等 ${ }^{[87]}$ 报道了另一种合成联-1,3,4-啞二唑的 方法: 利用联四唑 $(\mathbf{8 0})$ 与三氟酸酐反应得到 $5,5^{\prime}$-三氟甲 基-联-1,3,4-噁二唑 $(81), 173 \mathrm{~K}$ 下理论密度为 $1.980 \mathrm{~g} \bullet$ $\mathrm{cm}^{-3}$, 未报道其理论爆速(Eq. 25).

$2.43,3^{\prime}$-联-1,3,4-噁二唑-5,5'-二乙酯及其含能衍生物 合成

Yang 等 ${ }^{[88]}$ 以二氨基乙二肟和丙二酸二己酯为起始 反应原料，两步法合成 3,3'-联-1,3,4-噁二唑-5,5'-二乙酯 (84)(图 42).<smiles>O=C(OC(=O)C(F)(F)F)C(OC(=O)C(F)(F)F)C(F)(F)F</smiles>
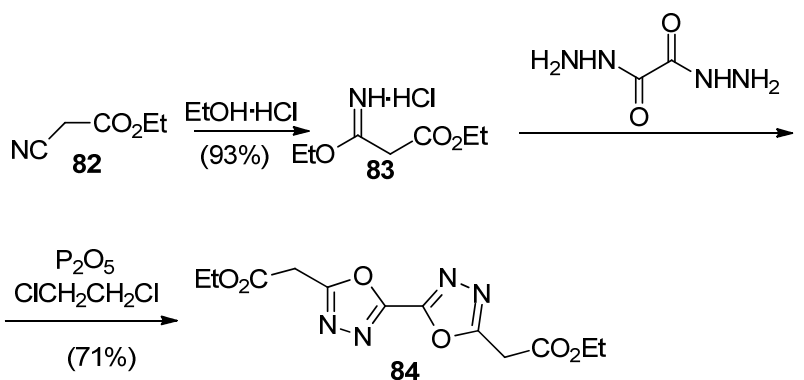

图 42 化合物 84 合成

Figure 42 Synthesis of compound 84

可能的反应机理为: 在酸性条件下，首先氰基 $\mathrm{N}$ 经 亲核进攻、共振得到 3-乙氧基-亚氨基丙酸乙酯盐酸盐 (图 43), 再经亲核反应、共振、脱去两分子的乙醇后生 成 3,3'-联 1,3,4-噁二唑-5,5'-二乙酯(图 44).

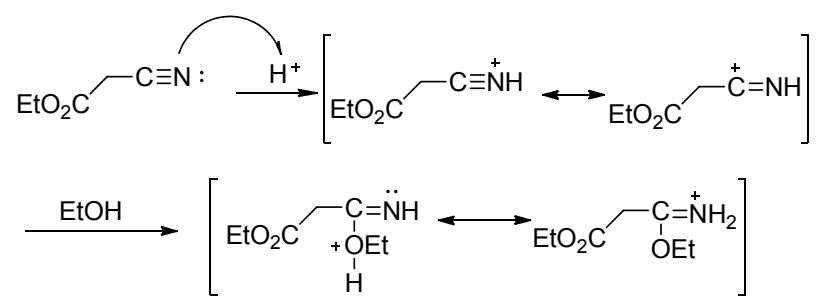

图 43 可能的成盐反应机理

Figure 43 Proposed reaction mechanism of salifying.

Yang 等 ${ }^{[88]}$ 以 84 为底物, 分别将其(1)经水解、硝化 合成了具有多硝基结构的 5,5'-二(三硝基甲基)-2,2'-联1,3,4-噁二唑 $(86)$; (2)经硝化、水解，与不同的富氮碱反 应生成含能离子盐 $88 \mathrm{a} \sim \mathbf{8 8 k}$. 这一系列化合物爆速为 $8770 \sim 7978 \mathrm{~m} \bullet \mathrm{s}^{-1}$, 其中羟胺盐 $(\mathbf{8 8 f})$ 的爆速为 $8770 \mathrm{~m} \bullet$ $\mathrm{s}^{-1}$, 密度为 $1.85 \mathrm{~g} \cdot \mathrm{cm}^{-3}, \mathrm{IS}=20 \mathrm{~J}, \mathrm{FS}=240 \mathrm{~N}$, 综合性 能与 RDX 相当(图 45).

\section{5 其它 1,3,4-噁二唑含能化合物}

由于 1,3,4-噁二唑的 2,5 位很不活泼(2-硝基-5-氨基1,3,4-噁二唑及 2,5-二硝基-1,3,4-噁二唑均尚未见报道), 因此人们考虑利用酯基 $\alpha$-碳的活泼性引入高能基团. Shreeve 等 ${ }^{[74]}$ 利用 1,3,4-啞二唑-2,5-二乙酸酯(89)的 $\alpha$-碳 引入硝基，通过硝化得到其二硝基产物(91)、三硝基产 物(92)及其 6 种含能盐 $(90 \mathrm{a} \sim 90 \mathrm{~h})$. 这也是目前报道的 唯一 1,3,4-噁二唑单环型含能化合物(图 46). 

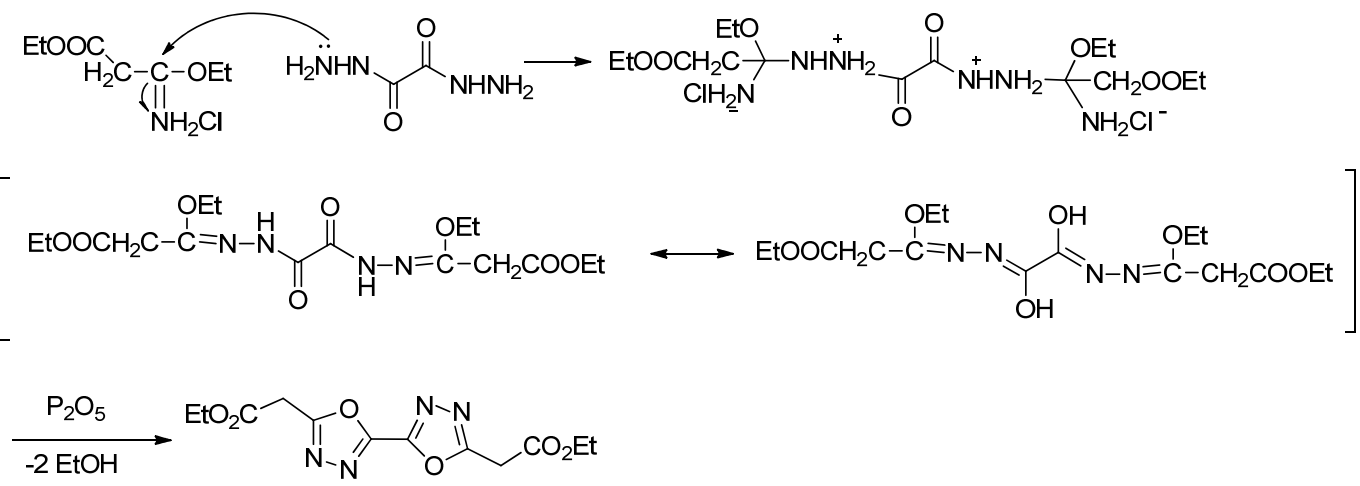

图 44 环化反应机理

Figure 44 Proposed cyclization reaction mechanism
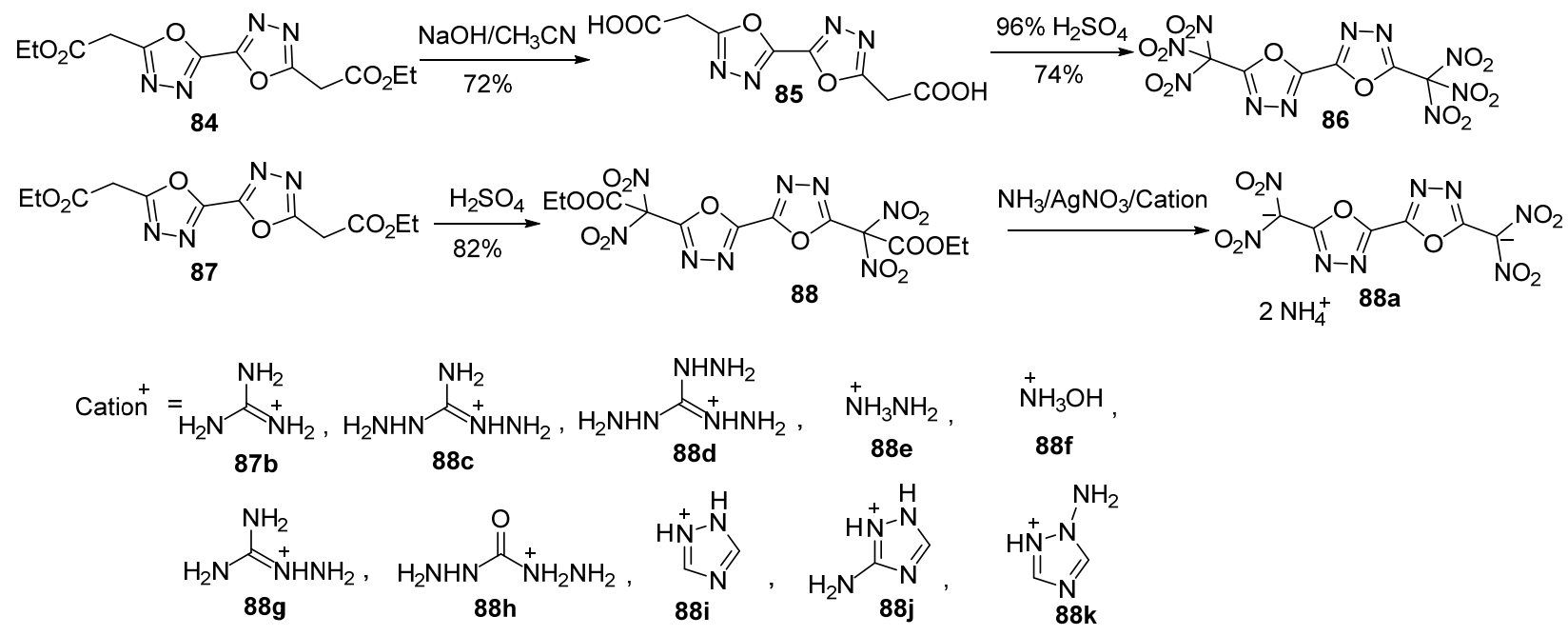

图 45 化合物 86 及含能离子盐 $88 \mathrm{a} \sim 88 \mathrm{k}$ 的合成

Figure 45 Synthesis of compound 86 and energetic ion salts $\mathbf{8 8 a} \sim \mathbf{8 8 k}$

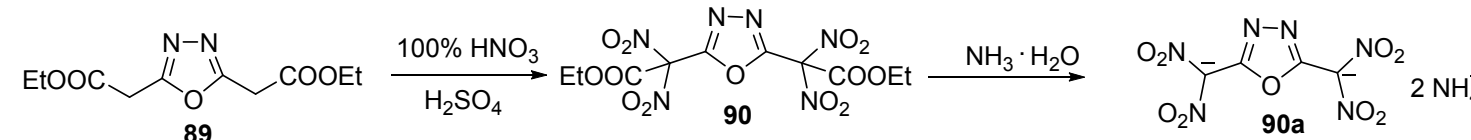

89 $\mathrm{H}_{2} \mathrm{SO}_{4}$
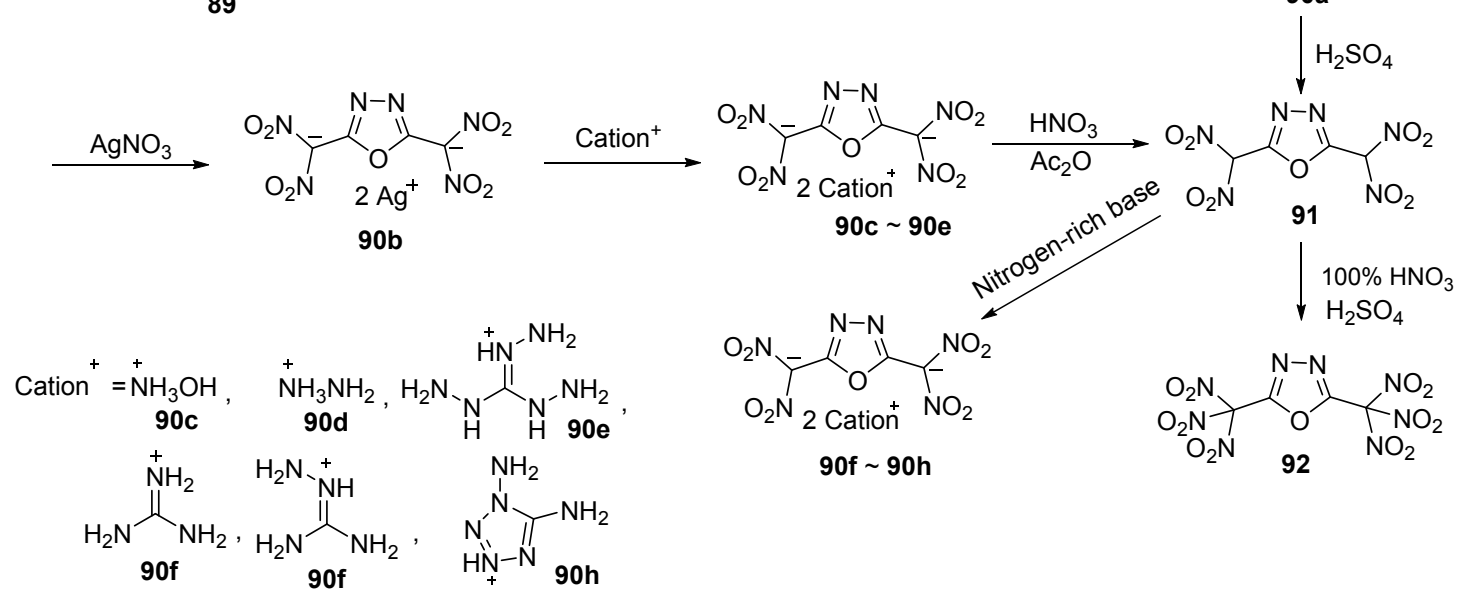

图 46 化合物 91 和 92 和含能离子盐的合成

Figure 46 Synthesis of compounds 91 and 92 and its energetic ion salts

Shreeve 等 ${ }^{[89]}$ 以 93 为原料，经硝化、碱性条件下脱 酯生成 4 种含能离子盐; 再氧化成酮得到化合物 96 . 这 
一系列化合物理论爆速为 $8042 \sim 8821 \mathrm{~m} \cdot \mathrm{s}^{-1}$, 其中羟胺 盐的爆速为 $8561 \mathrm{~m} \bullet \mathrm{s}^{-1}$, 密度为 $1.87 \mathrm{~g} \bullet \mathrm{cm}^{-3}, \mathrm{IS}=33 \mathrm{~J}$, $\mathrm{FS}=360 \mathrm{~N}$, 是一种新型钝感含能离子盐(图 47).

\subsection{1,3,4-噁二唑含能化合物性能研究}

与 1,2,4-噁二唑相比，1,3,4-噁二唑生成焓更低，且 1,3,4-噁二唑的 2,5 位很不活泼(如氨基很难氧化成硝基), 难以引入一些高能基团, 因此目前研究较少. 但是 1,3,4-噁二唑生成焓低, 含氧量高, Shreeve 课题组 ${ }^{[89]}$ 认 为它可能对呋咱衍生物具有很好的稳定作用.

虽然目前报道的 1,3,4-噁二唑基含能化合物并不丰 富, 但可以看到, 1,3,4-啞二唑环基含能化合物普遍感度 低, 有着可接受的密度和爆速, 并且已经出现一些有应 用潜力的含能化合物, 如 TKX-55 等为代表的熔铸型炸 药和以 69 为代表的高能炸药(表 2).

\section{3 结论与展望}

综上所述, 异呋咱类含能化合物的设计及合成已经 取得了一定的进展. 事实证明, 异呋咱环是优良的含能
化合物结构单元，可有效的降低含能化合物感度. 部分 异呋咱类含能化合物和含能离子盐表现出结构致密, 能 量水平高且感度低的特点, 特别是部分平面型异呋咱含 能化合物综合性能已优于 HMX，具有潜在应用价值.

氮杂环骨架的构建是含能材料合成的灵魂和核心. 迄今为止, 相对于 1,2,5-啞二唑及 1,2,4-啞二唑为母体结 构单元的含能化合物，1,3,4-噁二唑类含能化合物报道 相对较少, 这主要是由于 1,3,4-噁二唑的环化反应收率 受取代基影响较大，其次，其 2,5 位反应活性较差，进行 后续反应(如氨基偶氮化、氧化等)较为困难，制约了 1,3,4-噁二唑类含能化合物的发展, 如何高效构建 1,3,4啞二唑母体单元及在其邻位引入其它高氮杂环构建母 体骨架是今后 $1,3,4$-惡二唑类含能化合物研究的重点和 难点. 异呋咱类含能化合物研究才刚起步, 意味着该领 域发展空间很大. 随着含能化合物合成技术的不断进 步，有望涌现有工业化价值的新型异呋咱类不敏感含能 材料.<smiles>CCOC(=O)CO[N+](=O)[O-]</smiles><smiles>CC(C)(C)O[N+](=O)[O-]</smiles>

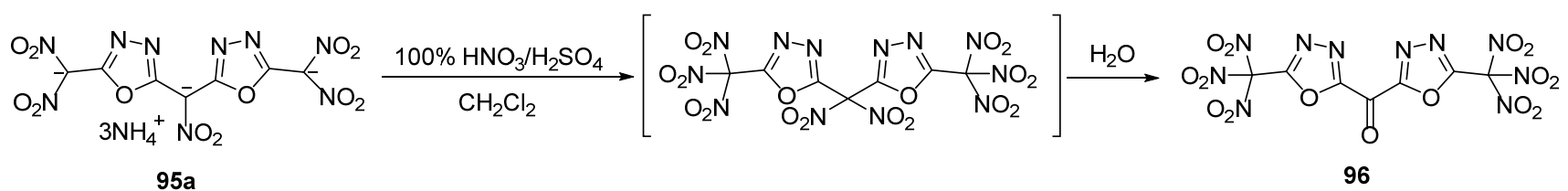

图 47 化合物 94 和 96 及含能离子盐的合成

Figure 47 Synthesis of compounds 94 and 96 and energetic ion salt

表 2 1,3,4-惡二唑化合物与 RDX 及 HMX 性能对比

Table 2 Properties of some 1,3,4-oxadiazole compounds compared with RDX and HMX

\begin{tabular}{|c|c|c|c|c|c|c|}
\hline Substrate & $\mathrm{FS} / \mathrm{N}$ & IS/J & $T_{\mathrm{dec}} /{ }^{\circ} \mathrm{C}$ & $p /\left(\mathrm{g} \cdot \mathrm{cm}^{-3}\right)$ & $V_{\mathrm{det}} /\left(\mathrm{m} \cdot \mathrm{s}^{-1}\right)$ & $H_{50} / \mathrm{cm}$ \\
\hline $\operatorname{RDX}^{[70]}$ & 120 & 7.4 & 210 & 1.86 & 8830 & 22 \\
\hline $\mathrm{HMX}^{[71]}$ & 120 & 7.0 & 260 & 1.91 & 9110 & - \\
\hline TKX-55 $5^{[21]}$ & $>360$ & 5 & 335 & 1.837 & 8030 & \\
\hline $69^{[72,73]}$ & $60^{[73]}, 72^{[72]}$ & $1^{[72]}, 5^{[72]}$ & $200^{[72]}, 210^{[73]}$ & $1.99^{[73]}$ & $9481^{[73]}$ & \\
\hline $69 \mathbf{a}^{[72]}$ & 360 & 10 & 197 & 1.95 & 9255 & \\
\hline $\mathbf{8 8 f}^{[88]}$ & 240 & 20 & 160.2 & 1.85 & 8770 & \\
\hline $90 \mathbf{c}^{[89]}$ & 360 & 20 & 146 & 1.89 & 9266 & \\
\hline $\mathbf{9 5} \mathbf{c}^{[90]}$ & 360 & 33 & 180 & 1.87 & 8561 & \\
\hline
\end{tabular}




\section{References}

[1] Zhang, J. H.; Zhang, Q. H.; Thao, T. V.; Parrish, D. A.; Shreeve, J. M. J. Am. Chem. Soc. 2015, 137, 1697.

[2] Zhang, J. H.; Shreeve, J. M. J. Phys. Chem. C 2015, 119, 12887.

[3] Zhang, J. H.; Shreeve, J. M. J. Am. Chem. Soc. 2014, 136, 4437.

[4] Klapötke, T. M.; Krumm, B.; Scherr, M.; Haiges, R.; Christe, K. O. Angew. Chem., Int. Ed. 2007, 46, 8686.

[5] Joo, Y. H.; Twamley, B.; Garg, S.; Shreeve, J. M. Angew. Chem., Int. Ed. 2008, 47, 6263.

[6] Zhang, J. R.; Bi, F. Q.; Lian, P.; Zhang, J. L.; Wang, B. Z. Chin. J. Org. Chem. 2016, 36, 1197 (in Chinese).

(张俊林, 肖川, 翟连杰, 王锡杰, 毕福强, 王伯周, 有机化学, 2016, 36, 1197.)

[7] Klapötke, T. M.; Mayr, N.; Stierstorfer, J.; Weyrauther, M. Chem. Eur. J. 2014, 20, 1410.

[8] Thottempudi, V.; Yin, P.; Zhang, J. H.; Parrish, D. A.; Shreeve, J. M. Chem. Eur. J. 2014, 20, 542.

[9] Veauthier, J. M.; Chavez, D. E.; Tappan, B. C.; Parrish, D. A. J. Energy Mater. 2010, 28, 229.

[10] He, J. X.; Lu, Y. H.; Lei, Q.; Cao Y. L. Chin. J. Explos. Propellants 2011, 34, 9 (in Chinese) (何金选，卢艳华，雷晴，曹一林，火炸药学报, 2011, 34, 9.)

[11] Sheremetev, A. B.; Ivanova, E. A.; Spiridonova, N. P.; Melnikova S. F.; Tselinsky I. V.; Suponitsky K. Y.; Antipin, M. Y. J. Heterocycl. Chem.

[12] Lim, C. H.; Kim, T. K.; Kim, K. H.; Chung, K. H.; Kim, J. S. Bull. Korean Chem. Soc. 2010, 31, 1400.

[13] Zhang, J. R.; Bi, F. Q.; Lian, P.; Zhang, J. L.; Wang, B. Z. Chin. J. Org. Chem. 2017, 37, 2736 (in Chinese) (张家荣, 毕福强, 廉鹏, 张俊林, 王伯周, 有机化学, 2017, 37, 2736.)

[14] Sheremetev, A. B.; Kharitonova, O. V.; Mantseva, E. V.; Kulagina, V. O.; Shatunova, E. V.; Aleksandrova, N. S.; Mel'nikova, T. M.; Ivanova, E. A.; Dmitriev, D. E.; Eman, V.; Yundin, I. L.; Kuz' min, V. S.; Strelenko, Y. A.; Novikova, T. S.; Lebedev, O. V.; Khmel'nitskii, L. I. Russ J. Org. Chem. 1999, 35, 1525.

[15] Ge, Z. X.; Lai, W. P.; Lian, P.; Wang, B. Z.; Xue, Y. Q. Chin. J. Explos. Propellants 2007, 30, 5 (in Chinese).

(葛忠学, 来蔚鹏, 廉鹏, 王伯周, 薛永强, 火炸药学报, 2007, 30, 5.)

[16] Sun, Q.; Shen, C.; Li, X.; Lin, Q. H.; Lu, M. J. Mater. Chem. A 2017, 5, 11063.

[17] Fu, Z. D.; Su, R.; Wang, Y.; Wang, Y. F.; Zeng, W.; Xiao, N.; Wu, Y. K.; Zhou, Z. M.; Chen, J.; Chen, F. X. Chem.-Eur. J. 2012, 18, 1886 .

[18] Korkin, A. A.; Bartlett, R. J. J. Am. Chem. Soc. 1996, 118, 12244

[19] Kamlet, M. J.; Jacobs, S. J. J. Chem. Phys. 1968, 48, 23.

[20] Qu, Y. Y.; Zeng, Q.; Wang, J.; Ma, Q.; Li, H. Z.; Li, H. N.; Yang, G. C. Chem.-Eur.J. 2016, 22, 12527.

[21] Klapötke, T. M.; Witkowski, T. G. ChemPlusChem 2016, 81, 357.

[22] Johnson, E. C.; Sabatini, J. J.; Chavez, D. E.; Sausa, R. C.; Byrd, E. F. C.; Wingard, L. A.; Guzmàn, P. E. Org. Process Res. Dev. 2018, 22,736

[23] Chavez, D.; Klapötke, T. M.; Parrish, D.; Piercey, D. G.; Stierstorfer, J. Propellants Explos. Pyrotech. 2014, 39, 641.

[24] Yu, Q.; Cheng, G. B.; Ju, X. H.; Lu, C. X.; Lin, Q. H.; Yang. H. W. New J. Chem. 2017, 41, 1202.

[25] Tamura, M.; Ise, Y.; Okajima Y.; Nishiwaki, N.; Ariga, M. Synthesis 2006, 3453.

[26] Tsiulin, P. A.; Sosnina, V. V.; Krasovskaya, G. G.; Danilova, A. S.; Baikov, S. V.; Kofanov, E. R. Russ. J. Org. Chem. 2011, 47, 1874.

[27] Eloy, F.; Lenaers, R. Chem. Rev. 1962, 62, 155.

[28] Tiemann, F.; Kruger, P. Chem. Ber. 1884, 17, 1658.

[29] Ooi, N. S.; Wilson, D. A. J. Chem. Soc., Perkin Trans. 2 1980, 1792.

[30] Chiou, S. S.; Shine, H. H. J. Heterocycl. Chem. 1989, 26, 125.

[31] Gangloff, R. A.; Litvak, J; Shelton, E. J.; Sperandio, D.; Wang, V.
R.; Rice, K. D. Tetrahedron Lett. 2001, 42, 1441

[32] Lukin, K.; Kishore, V. J. Heterocycl. Chem. 2014, 51, 256.

[33] Otaka, H.; Ikeda, J.; Tanaka, D.; Tobe, M. Tetrahedron Lett. 2014, 55,979 .

[34] Kaboudin, B.; Navaee, M. Heterocycles 2003, 60, 2287.

[35] Kiseleva, V. V.; Gakh, A. A.; Fainzil'berg, A. A. Bull. Acad. Sci. USSR, Div. Chem. Sci. 1991, 100, 1888.

[36] Huang, X. C.; Guo, T.; Wang, Z. J.; Liu, M.; Qiu, S. J.; Yao, B. J.; Li, H. L.; Jiang, J.; Tang, W.; Lv, Y. D.; Zhang, Y.; Chen, Z. Q.; Shi, Q.; Zheng, X. D. CN 106632124, 2017.

[37] Huang, X. C.; Wang, Z. J.; Guo, T.; Qin, M. N.; Liu, M.; Qiu, S. Chin. J. Energ. Mater. 2017, 25, 603 (in Chinese).

(黄晓川，王子俊，郭涛，秦明娜，刘敏，邱少君，含能材料, 2017, 25, 603.)

[38] Kettner, M. A.; Karaghiosoff, K; Klapötke, T. M.; Sućeska, M.; Wunder, S. Chem.-Eur. J. 2014, 20, 7622.

[39] Kettner, M. A.; Klapötke, T. M. Chem. Commun. 2014, 50, 2268.

[40] Gregory, G. I.; Warburton, W. K.; Seale, P. W. DE 2224338, 1972.

[41] Pagoria, P. F.; Zhang, M. X.; Zuckerman, N. B.; DeHope, A. J.; Parrish, D. A. Chem. Heterocycl. Compd. 2017, 53, 760.

[42] Dolbier, W. R.; Burkholder, C. R.; Médebielle, M. J. Fluorine Chem. 1999, 95, 127.

[43] Neves Filho, R. A. W.; Silva-Alves, D. C. B.; Anjos, J. V. D.; Srivastava, R. M. Synth. Commum. 2013, 43, 2596.

[44] Zhang C. J. Mol. Struc. Theochem 2006, 795, 77

[45] Dippold, A. A.; Izsák, D.; Klapötke, T. M. Chem. Eur. J. 2013, 19, 12042.

[46] Tsyshevsky, R.; Pagoria, P.; Zhang, M. X.; Racoveanu, A.; Parrish, D. A.; Smirnov, A. S.; Kuklja. M. M. J. Phys. Chem. C 2017, 121, 23853.

[47] Tarasenko, M.; Duderin, N.; Sharonova, T.; Baykov, S.; Shetnev, A.; Smirnov. V. A. Tetrahedron Lett. 2017, 58, 3672.

[48] Tadikonda, R.; Nakka, M.; Gajula, M. B.; Rayavarapu, S.; Gollamudi, P. R.; Vidavalur, S. Synth. Commum. 2014, 44, 1978.

[49] Klapçtke, T. M.; Piercey, D. G. Inorg. Chem. 2011, 50, 2732.

[50] Li, Y. C.; Qi, C.; Li, S. H.; Zhang, H. J.; Sun, C. H.; Yu, Y. Z.; Pang, S. P. J. Am. Chem. Soc. 2010, 132, 12172.

[51] Yin, P.; Parrish, D. A.; Shreeve, J. M. Chem.-Eur. J. 2014, 20, 6707.

[52] Shaposhnikov, S. D.; Korobov, N. V.; Sergievskii, A. V.; Pirogov, S. V.; Mel'nikova, S. F.; Tselinskii, I. V. Russ. J. Org. Chem. 2002, 38 , 1351.

[53] Andrianov, V. G.; Semenikhina, V. G.; Eremeev, A. V. J. Heterocycl. Compd. 1994, 30, 4

[54] Yan. C; Wang, K. C.; Liu, T. L.; Yang, H. W.; Cheng, G. B.; Zhang, Q. H. Dalton Trans. 2017, 46, 14210.

[55] Dimsdale, M. J. J. Heterocycl. Chem. 1981, 18, 37.

[56] Zhao, G.; He, C. L.; Yin, P.; Imler, G. H.; Parrish, D. A.; Shreeve, J. M. J. Am. Chem. Soc. 2018, 140, 3560.

[57] Shaposhnikov, S. D.; Korobov, N. V.; Sergievskii, A. V.; Pirogov, S. V.; MelÏnikova, S. F.; Tselinskii, I. V. Russ. J. Org. Chem. 2001, 38, 1351.

[58] Wei, H.; He, C. L.; Zhang, J. H.; Shreeve, J. M. Angew. Chem., Int. Ed. 2015, 54, 9367.

[59] Wang, Q.; Shao, Y. L.; Lu, M. Cryst. Growth Des. 2018, 18, 6150.

[60] Yarovenko, V. N.; Taralashvili, V. K.; Zavarzin, I. V.; Krayushkin, M. M. Tetrahedron 1990, 46, 3941.

[61] Augustine, J. K.; Akabote, V.; Hegde, S. G.; Alagarsamy, P. J. Org. Chem. 2009, 74, 5640.

[62] Huffman, K. R.; Schaefer, F. C. Org. Chem. 1963, 28, 1816.

[63] Huttunen, K. M.; Leppänen, J.; Kemppainen E.; Palonen, P.; Rautio, J.; Järvinen, T.; Vepsäläinen, J. Synthesis 2008, 3619.

[64] Thottempudi, V.; Zhang, J. H.; Hea, C. L.; Shreeve, J. M. RSC Adv. 2014, 4, 50361.

[65] Yuan, Y. B.; Nie, J.; Wang, S. J.; Zhang, Z. B. Chin. J. Org. Chem. 2005, 25, 394 (in Chinese). (袁余斌, 聂进, 王炼今, 张正波，有机化学, 2005, 25, 394.).

[66] Pang, F. Q.; Wang, G. L.; Lu, T.; Fan, G. J.; Chen, F. X. New J. Chem. 2018, 42, 4036. 
[67] Tang, Y. X.; Gao, H. X.; Mitchell, L. A.; Parrish, D. A.; Shreeve, J. M. Angew. Chem., Int. Ed. 2016, 55, 1147

[68] Yong, T. G.; Lin, M. Z.; Fu, Q. P.; Xiu, J. Q.; Jing, L. H.; Chen, F. X. Chin. Chem. Lett. 2016, 27, 433.

[69] Wang, G. L.; Lu, T.; Fan, G. J.; Li, C. Q.; Yin, H. Q.; Chen, F. X. Chem-Asian J. 2018, 13, 3718.

[70] Vroom, A. H.; Winkler, C. A. Can. J. Res. 1950, 28B, 701.

[71] Maycock, J. N.; Verneker, V. R.; Lochte, W. Phys. Status Solidi B $1969,35,849$.

[72] Hermann, T. S.; Karaghiosoff, K,; Klapötke, K. M.; Stierstorfer, J. Chem.-Eur. J. 2017, 23, 12087.

[73] Zhang, W. Q.; Zhang, J. H.; Deng, M. C.; Qi, X. J.; Nie, F.; Zhang, Q. H. Nat. Commun. 2017, 18, 1..

[74] Tang, Y. X.; He, C. L.; Mitchell, L. A.; Parrish, D. A.; Shreeve, J. M. J. Mater. Chem. A 2015, 3, 23143.

[75] Fang, T.; Tan, Q. T.; Ding, Z. W.; Liu, B. X.; Xu, B. Org. Lett. 2014, 16, 2342

[76] Si, Z. J.; Li, J.; Li, B.; Zhao, F. F.; Liu, S. Y.; Li, W. L. Inorg. Chem. 2007, 46, 6155

[77] Dufau, L.; Ressurreição, A. S. M.; Fanelli, R.; Kihal, N.; Vidu, A. Milcent, T.; Soulier, J. L.; Rodrigo, J.; Desvergne, A.; Leblanc, K.; Bernadat, G.; Crousse, B.; Ravaux, M. R.; Ongeri, S. J. Med. Chem. 2012, 55, 6762 .
[78] Balicki, R.; Nantka-Namirski, P. Acta Pol. Pharm. 1988, 45, 1.

[79] Saudi, M. N. S.; El-Semary, M. M. A.; Elbayaa, R. Y.; Jaeda, M. I.; Eissa, M. M.; Amer, E. I.; Baddour, N. M. Med. Chem. Res. 2012, $21,257$.

[80] Carlsen, P. H. J.; Jørgensen, K. B. J. Heterocycl. Chem. 1994, 31 , 805.

[81] Zhang, P.; Qu, S. N.; Wang, H. T.; Bai, B. L.; Li, M. Liq. Cryst. 2008, 35, 389 .

[82] Al-Talib, M.; Tastoush, H.; Odeh, N. Synth. Commun. 1990, 20, 1811.

[83] Isobe, T.; Ishikawa, T. J. Org. Chem. 1999, 64, 6989.

[84] James, C. A.; Poirier, B.; Grisé, C.; Martel, A.; Ruediger, E. H. Tetrahedron Lett. 2006, 47, 511 .

[85] Liras, S.; Allen, M. P.; Segelstein, B. E. Synth. Commun. 2000, 30, 437.

[86] Sharnin, G. P. Khim. Geterotsikl. 1977, 6, 741 .

[87] Kettner, M. A.; Klapötke, T. M.; Witkowski, T. G.; Hundling, F. V. Chem.-Eur. J. 2015, 21, 4238.

[88] Tian, J. W.; Xiong, H. L.; Lin, Q. H.; Cheng, G. B.; Yang, H. W. New J. Chem. 2017, 41, 1918.

[89] Yu, Q.; Imler, G. M.; Parrish, D. A.; Shreeve, J. M. Chem.-Eur. J. 2017, 23, 17682 